\title{
A Classic Herbal Formula Guizhi Fuling Wan for Menopausal Hot Flushes: From Experimental Findings to Clinical Applications
}

\author{
Mingdi Li ${ }^{1}{ }^{(\mathbb{D}}$, Andrew Hung ${ }^{2}$, Hong $\mathrm{Li}^{1}{ }^{1}$ and Angela Wei Hong Yang ${ }^{1, *}$ \\ 1 School of Health and Biomedical Sciences, RMIT University, Bundoora, VIC 3083, Australia \\ 2 School of Science, RMIT University, Melbourne, VIC 3001, Australia \\ * Correspondence: angela.yang@rmit.edu.au; Tel.: +61-3-9925-7175; Fax: +61-3-9925-7178
}

Received: 30 June 2019; Accepted: 15 August 2019; Published: 18 August 2019

\begin{abstract}
A classic herbal formula Guizhi Fuling Wan (GFW) has been used for managing menopausal hot flushes (MHFs), but the evidence across different study types has not been systematically summarized. This project investigated the clinical effects, phytochemistry, pharmacodynamics, and potential mechanisms of actions of GFW on the causative target proteins potentially driving MHFs. Twenty English and Chinese databases were searched for relevant clinical and experimental studies. A total of 12,988 studies were identified, of which 46 were included. Seven clinical studies demonstrated GFW had no statistically significant changes in the frequency and severity of MHFs; however, it could improve peripheral blood flow in the fingertips, jaw, and toes. Thirty-five studies on phytochemistry identified 169 chemical compounds of GFW. Four experimental studies revealed GFW's therapeutic effects (e.g., normalize calcitonin gene-related peptide (CGRP) level) and potential target protein/cytokine (estrogen receptor beta (ESR2) with genetic variation, CGRP receptor, and interleukin-8) on MHFs. Therapeutic effects across different study types were inconsistent, possibly due to the dose difference and genotype variety of ESR2 in the human population. Further clinical and experimental studies, as well as biochemical investigation on the mechanisms of actions of GFW, are recommended.
\end{abstract}

Keywords: Cinnamon twig and Poria cocos pill; menopause; literature review; herbal medicine; women's health; natural products; integrative medicine

\section{Introduction}

A menopausal hot flush (MHF) is a sudden and transient onset of erythema and warmth or burning sensation on the face and skin of the neck which appears during the transition to (pre-menopausal and peri-menopausal), and through, menopause (menopausal and post-menopausal) [1,2]. The onset commonly lasts for seconds to five minutes, but its persistence duration is unpredictable [3]. MHFs significantly impact the quality of life of both the sufferers and their families [3]. Its mechanism has not been fully investigated. The sudden reduction in estrogen level, the narrowed central thermoneutral zone, changes of the certain neurotransmitter synthesis (e.g., noradrenaline and serotonin), and peripheral vascular reactivity are linked to MHF onset [4-9]. Menopausal hormone therapy is the most popular and effective MHF management [10,11]. However, adverse effects, such as nausea, dizziness, dry mouth, and contraindication, with hormone-dependent diseases (e.g., breast cancer) exist [12] Thus, more than half of all middle-aged menopausal women have taken non-hormonal therapies [13], such as Chinese herbal medicine (CHM).

Guizhi Fuling Wan (GFW, also known as Keishibukuryogan) is a classic Chinese herbal formula that has been used for MHFs in modern clinical practice [14,15]. In ancient China, there was no specific 
terminology used for MHFs. One Classic book, Ying Er Lun (Treatise on Infants), advocated that GFW could be used to treat flushed complexion in females of which the description is similar to MHF symptoms. As recorded in Jin Gui Yao Lue Fang Lun (Synopsis of Prescriptions of the Golden Chamber) [16], GFW consists of five herbal ingredients, including Gui Zhi (Cinnamomi Ramulus), Shao Yao (Paeoniae Radix Alba or Paeoniae Radix Rubra), Mu Dan Pi (Moutan Cortex), Tao Ren (Persicae Semen), and Fu Ling (Poria) [16]. The 2015 edition of the Chinese Pharmacopeia indicates that Bai Shao (Paeoniae Radix Alba) is used in tablet and capsule form GFW, whereas Chi Shao (Paeoniae Radix Rubra) is used in honey pill form [17]. In 2006, a GFW product (capsule) was approved by the US Food and Drug Administration to enter Phase II clinical trials [18]. Recently, more experimental studies have been conducted to investigate the pharmacodynamic effects of GFW [19-21]. However, GFW's application and mechanisms of actions on MHFs have not been systematically reviewed. This paper investigated the therapeutic effects of GFW on the management of MHFs from clinical and experimental perspectives.

\section{Clinical Application of GFW on MHFs}

The formula name and its synonyms were searched as keywords in 20 electronic databases (Cochrane Library, PubMed, EMBASE, AMED, CINAHL, Informit, Science Direct, LILACS, ProQuest, Wiley Online Library, PsycINFO, IndMED, AcuBriefs, Ingenta, KoreaMed, ERIC, CNKI, CQVIP, Wanfang Data, and sinoMed) for English or Chinese language papers from the earliest records available in each database, up to April 2019. The reference lists of articles with relevant topics were manually screened to identify potentially relevant studies. After removal of duplicates, the titles and abstracts of identified potential studies were screened. Full-texts were obtained for eligibility assessment against the selection criteria. All types of clinical studies (including randomized controlled trials (RCTs), non-randomized parallel controlled studies, and case series) were considered for inclusion as long as they recruited patients with MHFs or used MHFs as one of the outcome measures. To ensure comparability of the intervention, only studies using GFW in non-modified forms (e.g., decoction, pills, granules, and capsules with acceptable variations of Bai Shao/Chi Shao/Shao Yao) were considered. For studies involving control groups, only those that compared GFW with placebo, no treatment, or conventional medicine were considered. Studies utilizing any co-interventions were excluded.

A predesigned form was used to record extracted data, including study design, setting, sample size, the age, and diagnosis of the participants, intervention, duration, and outcome measures (including frequency and severity of MHFs, level of hormones/cytokines, and peripheral blood flow). Corresponding authors of potential articles were contacted by email, Research Gate (www. researchgate.net), and LinkedIn (www.linkedin.com) for missing data. Replies with unpublished data were received from corresponding authors of two papers [22,23]. The quality of included studies was analyzed according to study types. For RCTs, the quality was assessed using Cochrane's risk of bias assessment tool [24] with focus on the following domains: random sequence generation (selection bias), allocation concealment (selection bias), blinding of participants and personnel (performance bias), blinding of outcome assessment (detection bias), incomplete outcome data (attrition bias), and selective reporting (reporting bias). Quality was categorized as "Low risk", "Unclear risk", and "High risk" of bias. The quality of parallel controlled studies was evaluated by ROBINS-I ("Risk Of Bias In Non-randomised Studies - of Interventions") [25], and the assessed domains included confounding, participant selection, intervention classification, deviations from intended interventions, missing data, outcome measurement, and reported results. Quality was classified as "Low risk", "Moderate risk", "Serious risk", and "Critical risk" of bias. The quality of case series was assessed in four domains according to the Instrument for Evaluating the Quality of case series in CHM, including study aims and design (two questions), descriptions of treatment protocol (three questions), descriptions of methods and therapeutic/side-effects (two questions), and conduct of the study (six questions) [26]. A score of "1" was given when 'Yes' was applied to one item. Any article with a total score $\geq 7$ was considered to have good quality. 
A total of 12988 records were identified, and seven of them met the inclusion criteria. Figure 1 illustrates the selection process of the included studies. Two RCTs [14,15], one parallel controlled study [27], and two case series [28,29] were included in this review. Another two studies [22,23] were claimed as controlled trials; however, two intervention groups were incomparable. Thus, the treatment groups of these two studies were considered as case series studies, and only data from GFW groups were extracted for analyses in this review. The characteristics of the included studies are summarized in Table 1.

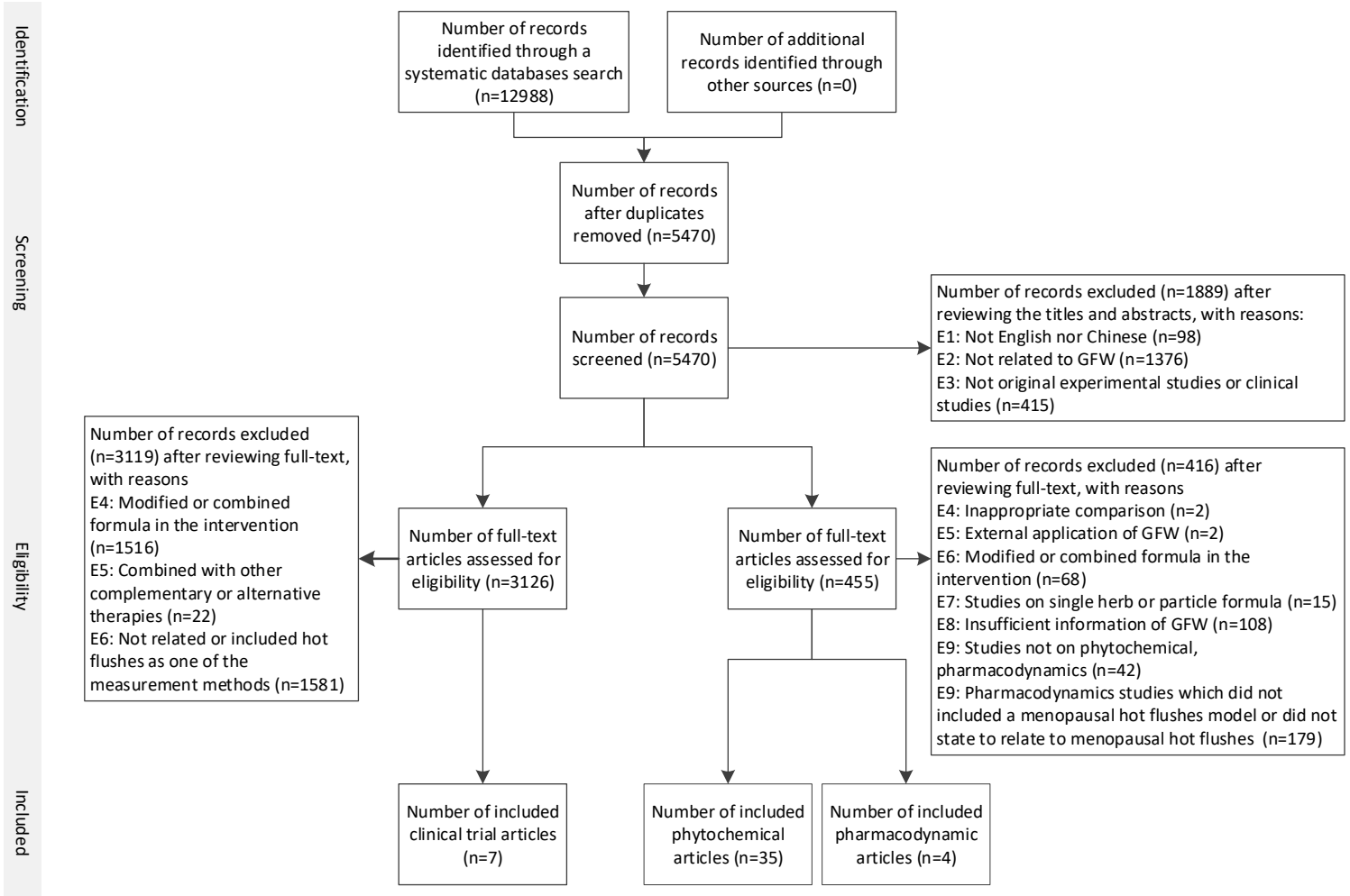

Figure 1. Flowchart of study selection procedures of Guizhi Fuling Wan on menopausal hot flushes. 
Table 1. Characteristics and quality assessment of included studies of Guizhi Fuling Wan for menopausal hot flushes.

\begin{tabular}{|c|c|c|c|c|c|c|c|}
\hline Study Name & Setting (Country) & Sample Size; Age & Diagnosis & Interventions & Duration & Outcomes Involved in this Review & Quality Assessment \\
\hline \multicolumn{8}{|c|}{ RCT } \\
\hline $\begin{array}{l}\text { Plotnikoff et al. } \\
\quad 2011[15]\end{array}$ & University (US) & $178 ; 45-58$ & Post-menopausal & $\begin{array}{l}\text { T1: GFW at } 7.5 \mathrm{~g} / \text { day }(n=62) ; \\
\text { T2: GFW at } 12.5 \mathrm{~g} / \text { day }(n=57) ; \\
\text { C: Placebo }(n=59)\end{array}$ & $\begin{array}{l}12 \text { weeks }(12 \\
\text { weeks duration }+ \\
1 \text {-week placebo } \\
\text { run-in period) }\end{array}$ & Frequency and severity of MHFs & $\mathrm{L}, \mathrm{L}, \mathrm{L}, \mathrm{L}, \mathrm{L}, \mathrm{H}$ \\
\hline $\begin{array}{l}\text { Ushiroyama et al. } \\
\quad 2005[14]\end{array}$ & College (Japan) & $\begin{array}{c}140 ; \\
\text { T: } 53.1 \pm 6.5 \\
\text { C: } 53.5 \pm 6.2\end{array}$ & Post-menopausal & $\begin{array}{c}\text { T: GFW } 7.5 \mathrm{~g} / \text { day }(n=70) ; \\
\text { C: oral HT (Premarin R } 0.625 \\
\text { mg/day + Provera R: } 2.5 \mathrm{mg} / \text { day) }(n \\
=70)\end{array}$ & 1 month & Blood flow in peripheral tissue & $\mathrm{U}, \mathrm{U}, \mathrm{H}, \mathrm{U}, \mathrm{H}, \mathrm{L}$ \\
\hline \multicolumn{8}{|c|}{ Parallel Controlled Study } \\
\hline $\begin{array}{l}\text { Yasui et al. } 2011 \\
{[27]}\end{array}$ & $\begin{array}{l}\text { Outpatient clinic of a } \\
\text { university hospital } \\
\text { (Japan) }\end{array}$ & $\begin{array}{c}77 ; \\
\text { T: } 52(39.3-58.7) \\
\text { C: } 51(43.4-57)\end{array}$ & $\begin{array}{c}\text { Pre-menopausal, } \\
\text { peri-menopausal, } \\
\text { post-menopausal, and } \\
\text { bilateral oophorectomized }\end{array}$ & $\begin{array}{l}\text { T: GFW } 7.5 \mathrm{~g} / \text { day }(n=38) ; \\
\text { C: No treatment }(n=39)\end{array}$ & 6 months & $\begin{array}{l}\text { The severity of MHFs, FSH, and } \mathrm{E}_{2} \\
\text { levels, IL-8, MCP-1, MIP-1 } \beta\end{array}$ & $\mathrm{L}, \mathrm{L}, \mathrm{L}, \mathrm{L}, \mathrm{M}, \mathrm{S}, \mathrm{L}$ \\
\hline \multicolumn{8}{|c|}{ Case Series } \\
\hline $\begin{array}{l}\text { Chen and Shiraki } \\
2003[28]\end{array}$ & Clinic (Japan) & $8 ; 53.59 \pm 0.58$ & Post-menopausal & GFW at $7.5 \mathrm{~g} /$ day $(n=8)$ & 4 weeks & Frequency of MHFs, plasma CGRP level & High-quality (score $=8$ ) \\
\hline $\begin{array}{l}\text { Namiki et al. } 2014 \\
\text { [29] }\end{array}$ & $\begin{array}{l}\text { Hospital and clinic of } \\
\text { university (Japan) }\end{array}$ & $\begin{array}{l}39 ; 49.5 \pm 4.69 \text { (for } \\
\quad 34 \text { patients) }\end{array}$ & $\begin{array}{l}\text { Post-menopausal }(n=18), \\
\text { 'not yet menopausal' }(n=21)\end{array}$ & GFW at $2.5 \mathrm{~g}, 3$ times/day $(n=39)$ & 12 weeks & Severity of MHFs, $\mathrm{FSH}$, and $\mathrm{E}_{2}$ levels & $\begin{array}{l}\text { High-quality (score }= \\
\text { 10) }\end{array}$ \\
\hline $\begin{array}{l}\text { Terauchi et al. } \\
2010 \text { [22] }\end{array}$ & $\begin{array}{l}\text { Hospital and clinic of } \\
\text { university (Japan) }\end{array}$ & $16 ; 51.1 \pm 2.4$ & Peri- and post-menopausal & Education + GFW $7.5 \mathrm{~g} /$ day $(n=16)$ & $144 \pm 58$ days & Frequency of MHFs & High-quality $($ score $=8)$ \\
\hline $\begin{array}{l}\text { Terauchi et al. } 2011 \\
\text { [23] }\end{array}$ & $\begin{array}{l}\text { Hospital and clinic of } \\
\text { university (Japan) }\end{array}$ & $30 ; 50.5 \pm 5.2$ & Peri-and post-menopausal & Education + GFW $7.5 \mathrm{~g} /$ day $(n=30)$ & $182 \pm 76$ days & Frequency of MHFs & High-quality (score $=7$ ) \\
\hline
\end{tabular}
university (Japan) low risk; M: moderate risk; RCT: randomized controlled trial; S: serious risk; T: treatment group; U: unclear risk. Quality of RCTs was assessed Cochrane's risk of bias assessment tool [24], quality of parallel controlled studies was evaluated by ROBINS-I ("Risk Of Bias In Non-randomised Studies - of Interventions") [25], and case series were assessed according to the Instrument for Evaluating the Quality of case series in Chinese herbal medicine [26]. 


\subsection{RCTs}

One RCT [15] reported that both low dosage (7.5 g/day) and high dosage (12.5 g/day) GFW could significantly reduce MHF frequency and severity when comparing between before and after the 12 -week intervention. When compared to placebo, there was no statistical significance. However, the reduction may be dose-dependent, as the results from the high dosage group demonstrated a greater reduction, which provided a direction for future research on the relationship between GFW dosage and clinical effects. Another RCT [14] reported that the peripheral blood flow in postmenopausal females with MHFs in the GFW group ( $n=67)$ was significantly decreased compared to the menopausal hormone therapy group $(n=64)$ under the jaw (mean difference (MD) $-3.56,95 \%$ confidence interval (CI) -5.17 to -1.95 ) and in the middle fingertip (MD $-7.10,95 \% \mathrm{CI}-10.99$ to -3.21 ) at the end of one-month treatment. Opposite effects on the blood flow in the third toe were observed in two groups: GFW increased the blood flow in the toe, whereas menopausal hormone therapy caused a decrease.

\subsection{Parallel Controlled Study}

The parallel controlled study [27] reported positive results, concluding that the severity of MHFs in $73.7 \%$ of the participants improved from severe to mild or moderate after six-month GFW intake. It was significantly higher than the control group. No significant difference in estradiol $\left(E_{2}\right)$ and follicle-stimulating hormone (FSH) levels was observed between groups. Results of serum cytokine level indicated that serum monocyte chemotactic protein-1 (MCP-1) level in women treated with keishibukuryogan decreased significantly $(-16.3 \%)$ compared to the no treatment group $(3.8 \%)$. Further analysis of the GFW responder group $(n=28)$ indicated that concentrations of serum interleukin (IL)-8 and macrophage inflammatory protein (MIP)- $1 \beta$ were reduced significantly, whereas those in the non-responders were increased dramatically. Sub analysis results on menopausal transition status showed that GFW decreased the IL-8 and MIP-1 $\beta$ levels in perimenopausal GFW responders and MCP-1 in postmenopausal GFW responders.

\subsection{Case Series}

One case series study [29] revealed that the improvement of MHF severity was related to genetic variation of the polymorphic dinucleotide (CA) repeat of the estrogen receptor beta (ESR2) gene on chromosome 14, which include three types: two short alleles (SS), two long alleles (LL), and a short and long allele (SL) [30,31]. MHF severity in participants with LL genotype improved significantly after the intervention. FSH level changes also depended on genotype: FSH levels decreased in participants with SS genotype but increased in participants with SL or LL genotypes. $\mathrm{E}_{2}$ level decreased in all genotype groups. Another case series [28] reported that the plasma calcitonin gene-related peptide (CGRP) level in eight post-menopausal participants significantly decreased after the four-week GFW intervention when compared to baseline data (MD $-2.88,95 \% \mathrm{Cl}-4.07$ to -1.69 ). CGRP is a potent vasodilator neuropeptide, and it is hypothesized to be a specific vasodilation neuropeptide of MHFs, which significantly rose in concentration during MHF onset [32]. However, GFW's effects on changes of frequency before and after intervention in three case series had no statistically significant difference (standardized mean difference (SMD) $-1.15,95 \%$ CI -2.49 to 0.20$)[22,23,28]$.

\section{Experimental Studies on Phytochemistry and Pharmacodynamics of GFW on MHFs}

We searched the 20 English and Chinese databases, detailed in Section 2, to identify experimental studies that have investigated the chemical compounds of GFW. Studies were excluded if they did not provide the following details: (1) plants with valid plant specimen voucher numbers, ingredients and ratios for raw herbs (decoction), or (2) company names and batch/lot numbers for purchased patent products (pills, granules, or capsules).

For pharmacodynamics, the same set of 20 databases was searched to identify experimental studies that have investigated GFW on MHFs. To be eligible to be included, the studies must provide 
the details as specified in (1) and (2) as above. Also, experiments were required to have (3) utilized cell or animal models related to MHFs; (4) compared GFW's effects with the blank model control group (i.e., no treatment); (5) evaluated the pharmacodynamic effects on MHFs. Studies involving any co-interventions were excluded.

The structures of chemical compounds were drawn using BIOVIA Draw 2019 [33] or Avogadro [34]/Discovery Studio Visualizer 2019 [35]. Molecular weight was calculated by Lenntech Molecular Weight Calculator (https://www.lenntech.com/calculators/molecular/molecular-weightcalculator.htm). The quality of in vivo studies was assessed based on 20 domains specified in the Animal Research: Reporting of In Vivo Experiments (ARRIVE) guidelines [36].

As a result, 35 studies on phytochemistry, and four studies on pharmacodynamics met the inclusion criteria. Figure 1 illustrates the selection process of the included studies.

\subsection{Phytochemistry of GFW}

The included studies on phytochemicals from GFW reported 169 compounds: four from Cinnamomi Ramulus (A1 and B1-B3), 13 from Persicae Semen (A1 and C1-C12), 41 from Moutan Cortex (A1, D1-D20, and E1-E20), 31 from Paeoniae Radix (A1, D1-D20, and F1-F10), 31 from Poria (A1 and G1-G30), 44 without clearly defined resources (H1-H44), and 29 inorganic elements. Table 2 outlines the characteristics of chemical constituents isolated from GFW. The molecular structures of 144 phytochemicals could be identified with their corresponding PubChem CIDs (compound identification number)/SIDs (substance identification number), whereas 25 constituents were not available in the PubChem database. Among those 25 chemical components, structures of 23 phytochemicals (C3, C6, D1, D6, D10, D12, D13, D14, D19, D20, E1, E10, F1, F2, F7, G4, G5, G12, G14, G21, H1, H2, H15) were provided in the included studies. One compound structure (G10) was available in a reference paper (as cited in Table 2), whereas one (G11) could not be found in any known data source. Details of the 24 molecular structures are presented in Figure 2.

The latest standards for different GFW forms established by high-performance liquid chromatography (HPLC) to guarantee the quality of GFW were cinnamic acid (B2) $\geq 72 \mu \mathrm{g}$ and paeonol (E14) $\geq 6 \mathrm{mg}$ per pill in honey pill form; paeonol (E14) $\geq 1.8 \mathrm{mg}$, paeoniflorin (F9) $\geq 3 \mathrm{mg}$, and amygdalin (C2) $\geq 1.5 \mathrm{mg}$ per tablet in tablet form; paeonol (E14) $\geq 1.8 \mathrm{mg}$, paeoniflorin (F9) $\geq 3 \mathrm{mg}$, and amygdalin (C2) $\geq 0.9 \mathrm{mg}$ per capsule in capsule form [17]. 
Table 2. Chemical compounds from Guizhi Fuling Wan.

\begin{tabular}{|c|c|c|c|c|c|c|}
\hline ID & List of Chemical Compounds & Form of GFW & Molecular Formula & Molecular Weight (g/mol) & PubChem CID/SID & Methods \\
\hline A & \multicolumn{6}{|c|}{ All ingredients $(n=1)$} \\
\hline A1 & $\begin{array}{l}\text { Sucrose/isomer (isomer only in } \\
\text { Paeoniae radix) }\end{array}$ & Capsule & $\mathrm{C} 12 \mathrm{H} 22 \mathrm{O} 11$ & 342.297 & 5988 & LC-MS/UPLC-ESI-Q-TOF-MS [37] \\
\hline B & \multicolumn{6}{|c|}{ Cinnamomi Ramulus $(n=3)$} \\
\hline B1 & Cinnamaldehyde & Capsule, pill & $\mathrm{C} 9 \mathrm{H} 8 \mathrm{O}$ & 132.162 & 637511 & HPLC [38,39], SPE-HPLC [40], UPLC [41] \\
\hline B2 & Cinnamic acid & Capsule, pill & $\mathrm{C} 9 \mathrm{H} 8 \mathrm{O} 2$ & 148.161 & 444539 & $\begin{array}{l}\text { HPLC [38,39], multiple chromatographic } \\
\text { methods [42], HPLC-ESI-Q-TOF/MS [43], } \\
\text { DGLC [44], UPLC [41], HPLC-MS/MS [45] }\end{array}$ \\
\hline B3 & Protocatechuic acid & Capsule & $\mathrm{C} 7 \mathrm{H} 6 \mathrm{O} 4$ & 154.121 & 72 & $\begin{array}{l}\text { LC-MS/UPLC-ESI-Q-TOF-MS [37], multiple } \\
\text { chromatographic methods [42] }\end{array}$ \\
\hline $\mathrm{C}$ & \multicolumn{6}{|c|}{ Persicae Semen $(n=12)$} \\
\hline $\mathrm{C} 1$ & A-Linolenic acid & & $\mathrm{C} 18 \mathrm{H} 30 \mathrm{O} 2$ & 278.436 & 5280934 & HPLC [46] \\
\hline $\mathrm{C} 2$ & Amygdalin & Capsule, tablet & C20H27NO11 & 457.432 & 656516 & $\begin{array}{l}\text { LC-MS/UPLC-ESI-Q-TOF-MS [37], HPLC } \\
\text { [47-51], HPLC-ESI-Q-TOF/MS [43], DGLC } \\
\text { [44], UPLC [41], HPLC-MS/MS [45] }\end{array}$ \\
\hline C3 & Benzol- $\beta$-gentiobioside & Capsule & $\mathrm{C} 19 \mathrm{H} 28 \mathrm{O} 11$ & 432.42 & NF (Figure 2) & LC-MS/UPLC-ESI-Q-TOF-MS [37] \\
\hline C4 & Betulinic acid & Capsule & $\mathrm{C} 30 \mathrm{H} 48 \mathrm{O} 3$ & 456.711 & 64971 & LC-MS/UPLC-ESI-Q-TOF-MS [37] \\
\hline C5 & Linoleic acid & Capsule & $\mathrm{C} 18 \mathrm{H} 32 \mathrm{O} 2$ & 280.452 & 5280450 & LC-MS/UPLC-ESI-Q-TOF-MS [34], HPLC [46] \\
\hline C6 & $\begin{array}{l}\text { Mandelic acid } \\
\text { gentiobioside/isomer }\end{array}$ & Capsule & $\mathrm{C} 20 \mathrm{H} 28 \mathrm{O} 13$ & 476.43 & NF (Figure 2) & LC-MS/UPLC-ESI-Q-TOF-MS [37] \\
\hline C7 & $\begin{array}{l}\text { Mandelic } \\
\text { acid- } \beta \text {-D-glucopyranoside }\end{array}$ & Capsule & $\mathrm{C} 14 \mathrm{H} 18 \mathrm{O} 8$ & 314.29 & 73229629 & LC-MS/UPLC-ESI-Q-TOF-MS [37] \\
\hline $\mathrm{C} 8$ & Oleic acid & Capsule & $\mathrm{C} 18 \mathrm{H} 34 \mathrm{O} 2$ & 282.468 & 445639 & LC-MS/UPLC-ESI-Q-TOF-MS [37], HPLC [46] \\
\hline C9 & Palmitic acid & Capsule & $\mathrm{C} 16 \mathrm{H} 32 \mathrm{O} 2$ & 256.43 & 985 & LC-MS/UPLC-ESI-Q-TOF-MS [37] \\
\hline $\mathrm{C} 10$ & Prunasin & Capsule & $\mathrm{C} 14 \mathrm{H} 17 \mathrm{NO} 6$ & 295.291 & 119033 & LC-MS/UPLC-ESI-Q-TOF-MS [37] \\
\hline C11 & Stearic acid & Capsule & $\mathrm{C} 18 \mathrm{H} 36 \mathrm{O} 2$ & 284.484 & 5281 & LC-MS/UPLC-ESI-Q-TOF-MS [37] \\
\hline $\mathrm{C} 12$ & Trametenolic acid & Capsule & $\mathrm{C} 30 \mathrm{H} 48 \mathrm{O} 3$ & 456.711 & 12309443 & LC-MS/UPLC-ESI-Q-TOF-MS [37] \\
\hline
\end{tabular}


Table 2. Cont.

\begin{tabular}{|c|c|c|c|c|c|c|}
\hline ID & List of Chemical Compounds & Form of GFW & Molecular Formula & Molecular Weight (g/mol) & PubChem CID/SID & Methods \\
\hline D & \multicolumn{6}{|c|}{ Paeoniae Radix + Moutan Cortex $(n=20)$} \\
\hline D1 & 4'-O-galloypaeoniflorin & Capsule & $\mathrm{C} 30 \mathrm{H} 32 \mathrm{O} 15$ & 632.56 & NF (Figure 2) & LC-MS/UPLC-ESI-Q-TOF-MS [37] \\
\hline D2 & 4-hydroxybenzoic acid & Capsule & C7H6O3 & 138.122 & 135 & $\begin{array}{l}\text { LC-MS/UPLC-ESI-Q-TOF-MS [37], multiple } \\
\text { chromatographic methods [42] }\end{array}$ \\
\hline D3 & 4-O-galloylalbiflorin & Capsule & $\mathrm{C} 30 \mathrm{H} 32 \mathrm{O} 15$ & 632.571 & 135397096 & LC-MS/UPLC-ESI-Q-TOF-MS [37] \\
\hline D4 & Albiflorin & Capsule & $\mathrm{C} 23 \mathrm{H} 28 \mathrm{O} 11$ & 480.466 & 51346141 & $\begin{array}{l}\text { LC-MS/UPLC-ESI-Q-TOF-MS [37], RP-HPLC } \\
\text { [52], chromatography on silica gel, Sephdex } \\
\text { LH-20 columns and prep-HPLC-NMR [53], } \\
\text { HPLC-ESI-Q-TOF/MS [43], HPLC-MS/MS [45] }\end{array}$ \\
\hline D5 & Benzoylpaeoniflorin/isomer & Capsule & $\mathrm{C} 30 \mathrm{H} 32 \mathrm{O} 12$ & 584.574 & 21631106 & $\begin{array}{c}\text { chromatography on silica gel, Sephdex LH-20 } \\
\text { columns and prep-HPLC-NMR [53], } \\
\text { HPLC-ESI-Q-TOF/MS [43] }\end{array}$ \\
\hline D6 & Benzoylpaeoniflorin sulfate & Capsule & С $30 \mathrm{H} 32 \mathrm{O} 14 \mathrm{~S}$ & 648.63 & NF (Figure 2) & LC-MS/UPLC-ESI-Q-TOF-MS [37] \\
\hline D7 & Desbenzoylalbiflorin/ isomer & Capsule & $\mathrm{C} 16 \mathrm{H} 24 \mathrm{O} 10$ & 376.358 & 71452333 & LC-MS/UPLC-ESI-Q-TOF-MS [37] \\
\hline D8 & Digalloylglucose & Capsule & $\mathrm{C} 20 \mathrm{H} 20 \mathrm{O} 14$ & 484.366 & 129628549 & LC-MS/UPLC-ESI-Q-TOF-MS [37] \\
\hline D9 & Gallic acid & Capsule & C7H6O5 & 170.12 & 370 & $\begin{array}{c}\text { LC-MS/UPLC-ESI-Q-TOF-MS [37], RP-HPLC } \\
\text { [52], HPLC-ESI-Q-TOF/MS [43], UPLC [41], } \\
\text { HPLC-MS/MS [45] }\end{array}$ \\
\hline D10 & Galloyglucose/isomer & Capsule & $\mathrm{C} 13 \mathrm{H} 16 \mathrm{O} 10$ & 332.26 & NF (Figure 2) & LC-MS/UPLC-ESI-Q-TOF-MS [37] \\
\hline D11 & Galloyl paeoniflorin & Capsule & С $30 \mathrm{H} 32 \mathrm{O} 15$ & 632.571 & 494717 & $\begin{array}{c}\text { LC-MS/UPLC-ESI-Q-TOF-MS [37], } \\
\text { chromatography on silica gel and polydextran } \\
\text { gel columns and prep-HPLC-NMR/MS [54], } \\
\text { HPLC-ESI-QTOF/MS [43] }\end{array}$ \\
\hline D12 & Galloylpaeoniflorin sulfonate & Capsule & С $30 \mathrm{H} 32 \mathrm{O} 17 \mathrm{~S}$ & 696.63 & NF (Figure 2) & LC-MS/UPLC-ESI-Q-TOF-MS [37] \\
\hline D13 & Hexagalloyl glucose & Capsule & C48H36O30 & 1092.78 & NF (Figure 2) & LC-MS/UPLC-ESI-Q-TOF-MS [37] \\
\hline D14 & Isoxypaeoniflora & Capsule & $\mathrm{C} 23 \mathrm{H} 28 \mathrm{O} 12$ & 496.46 & NF (Figure 2) & LC-MS/UPLC-ESI-Q-TOF-MS [37] \\
\hline D15 & Methyl gallate & Capsule & C8H8O5 & 184.147 & 7428 & LC-MS/UPLC-ESI-Q-TOF-MS [37] \\
\hline D16 & Methyl vanillate & Capsule & $\mathrm{C} 9 \mathrm{H} 10 \mathrm{O} 4$ & 182.175 & 19844 & LC-MS/UPLC-ESI-Q-TOF-MS [37] \\
\hline D17 & Oxypaeoniflorin & Capsule & $\mathrm{C} 23 \mathrm{H} 28 \mathrm{O} 12$ & 496.465 & 21631105 & $\begin{array}{l}\text { LC-MS/UPLC-ESI-Q-TOF-MS [37], } \\
\text { chromatography on silica gel, Sephdex LH-20 } \\
\text { columns and prep-HPLC-NMR [53], } \\
\text { chromatography on silica gel, Sephdex LH-20 } \\
\text { columns, D-101 macroporous resin column, } \\
\text { and reverse RP-18 column and } \\
\text { HPLC-NMR/MS [55] }\end{array}$ \\
\hline
\end{tabular}


Table 2. Cont.

\begin{tabular}{|c|c|c|c|c|c|c|}
\hline ID & List of Chemical Compounds & Form of GFW & Molecular Formula & Molecular Weight (g/mol) & PubChem CID/SID & Methods \\
\hline D18 & Pentagalloylglucose & Capsule & $\mathrm{C} 41 \mathrm{H} 32 \mathrm{O} 26$ & 940.681 & 65238 & $\begin{array}{l}\text { LC-MS/UPLC-ESI-Q-TOF-MS [37], } \\
\text { HPLC-ESI-Q-TOF/MS [43] }\end{array}$ \\
\hline D19 & Tetragalloy glucose & Capsule & $\mathrm{C} 34 \mathrm{H} 28 \mathrm{O} 22$ & 788.57 & NF (Figure 2) & LC-MS/UPLC-ESI-Q-TOF-MS [37] \\
\hline D20 & Trigalloy glucose & Capsule & $\mathrm{C} 27 \mathrm{H} 24 \mathrm{O} 18$ & 636.47 & NF (Figure 2) & LC-MS/UPLC-ESI-Q-TOF-MS [37] \\
\hline E & \multicolumn{6}{|c|}{ Moutan Cortex $(n=20)$} \\
\hline E1 & 3-hydroxypaeonol & Capsule & C9H10O4 & 182.17 & NF (Figure 2) & LC-MS/UPLC-ESI-Q-TOF-MS [37] \\
\hline E2 & Apiopaeonoside & Capsule & $\mathrm{C} 2 \mathrm{OH} 28 \mathrm{O} 12$ & 460.432 & 127509 & LC-MS/UPLC-ESI-Q-TOF-MS [37] \\
\hline E3 & Benzoyloxypaeoniflorin & Capsule & $\mathrm{C} 30 \mathrm{H} 32 \mathrm{O} 13$ & 600.573 & 21631107 & $\begin{array}{l}\text { LC-MS/UPLC-ESI-Q-TOF-MS [37], } \\
\text { HPLC-ESI-Q-TOF/MS [43] }\end{array}$ \\
\hline E4 & Galloyloxypaeoniflorin & Capsule & $\mathrm{C} 30 \mathrm{H} 32 \mathrm{O} 16$ & 648.56 & 3036133 & LC-MS/UPLC-ESI-Q-TOF-MS [37] \\
\hline E5 & Mudanpioside A & & С $31 \mathrm{H} 34 \mathrm{O} 13$ & 614.6 & 21631101 & $\begin{array}{l}\text { Chromatography on silica gel and } \\
\text { polydextran gel columns and } \\
\text { prep-HPLC-NMR/MS [54], } \\
\text { HPLC-ESI-O-TOF/MS [43] }\end{array}$ \\
\hline E6 & Mudanpioside B & Capsule & С $31 \mathrm{H} 34 \mathrm{O} 14$ & 630.599 & 21631102 & LC-MS/UPLC-ESI-Q-TOF-MS [37] \\
\hline E7 & Mudanpioside C & Capsule & $\mathrm{C} 30 \mathrm{H} 32 \mathrm{O} 13$ & 600.573 & 21631098 & $\begin{array}{c}\text { LC-MS/UPLC-ESI-Q-TOF-MS [37], } \\
\text { chromatography on silica gel and polydextran } \\
\text { gel columns and prep-HPLC-NMR/MS [54], } \\
\text { HPLC-ESI-QTOF/MS [43] }\end{array}$ \\
\hline E8 & Mudanpioside D & Capsule & $\mathrm{C} 24 \mathrm{H} 30 \mathrm{O} 12$ & 510.492 & 21631103 & LC-MS/UPLC-ESI-Q-TOF-MS [37] \\
\hline E9 & Mudanpioside E & Capsule & $\mathrm{C} 24 \mathrm{H} 30 \mathrm{O} 13$ & 526.491 & 21631104 & LC-MS/UPLC-ESI-Q-TOF-MS [37] \\
\hline E10 & Mudanpioside E sulfonate & Capsule & C24H30O14S & 574.55 & NF (Figure 2) & LC-MS/UPLC-ESI-Q-TOF-MS [37] \\
\hline E11 & Mudanpioside F & Capsule & $\mathrm{C} 16 \mathrm{H} 24 \mathrm{O} 8$ & 344.36 & 21631108 & $\begin{array}{c}\text { LC-MS/UPLC-ESI-Q-TOF-MS [37], } \\
\text { chromatography on silica gel, Sephdex LH-20 } \\
\text { columns, D-101 macroporous resin column, } \\
\text { and reverse RP-18 column and } \\
\text { HPLC-NMR/MS [55] }\end{array}$ \\
\hline E12 & Mudanpioside $\mathrm{H} /$ isomer & Capsule & $\mathrm{C} 30 \mathrm{H} 32 \mathrm{O} 14$ & 616.572 & 71457654 & LC-MS/UPLC-ESI-Q-TOF-MS [37] \\
\hline E13 & Mudanpioside J & Capsule & $\mathrm{C} 31 \mathrm{H} 34 \mathrm{O} 14$ & 630.599 & 21593828 & LC-MS/UPLC-ESI-Q-TOF-MS [37] \\
\hline E14 & Paeonol & Capsule, pill, tablet & $\mathrm{C} 9 \mathrm{H} 10 \mathrm{O} 3$ & 166.176 & 11092 & $\begin{array}{c}\text { LC-MS/UPLC-ESI-Q-TOF-MS [37], HPLC } \\
\text { [38,39,47,50,51], gas chromatography [56], } \\
\text { RP-HPLC [57,58], SPE-HPLC [40], } \\
\text { HPLC-ESI-QTOF/MS [43], DGLC [44], UPLC } \\
\text { [41], HPLC-MS/MS [45] }\end{array}$ \\
\hline E15 & Paeonolide & Capsule & $\mathrm{C} 20 \mathrm{H} 28 \mathrm{O} 12$ & 460.432 & 442923 & LC-MS/UPLC-ESI-Q-TOF-MS [37] \\
\hline E16 & Paeonoside & Capsule & $\mathrm{C} 15 \mathrm{H} 20 \mathrm{O} 8$ & 328.317 & 53384347 & LC-MS/UPLC-ESI-Q-TOF-MS [37] \\
\hline
\end{tabular}


Table 2. Cont

\begin{tabular}{|c|c|c|c|c|c|c|}
\hline ID & List of Chemical Compounds & Form of GFW & Molecular Formula & Molecular Weight (g/mol) & PubChem CID/SID & Methods \\
\hline E17 & Suffruticoside A & Capsule & $\mathrm{C} 27 \mathrm{H} 32 \mathrm{O} 16$ & 612.537 & 9986231 & LC-MS/UPLC-ESI-Q-TOF-MS [37] \\
\hline E18 & Suffruticoside B & Capsule & $\mathrm{C} 27 \mathrm{H} 32 \mathrm{O} 16$ & 612.537 & 10258205 & LC-MS/UPLC-ESI-Q-TOF-MS [37] \\
\hline E19 & Suffruticoside C & Capsule & $\mathrm{C} 27 \mathrm{H} 32 \mathrm{O} 16$ & 612.537 & 10258206 & LC-MS/UPLC-ESI-Q-TOF-MS [37] \\
\hline E20 & Suffruticoside D & Capsule & $\mathrm{C} 27 \mathrm{H} 32 \mathrm{O} 16$ & 612.537 & 5321547 & LC-MS/UPLC-ESI-Q-TOF-MS [37] \\
\hline $\mathbf{F}$ & \multicolumn{6}{|c|}{ Paeoniae Radix $(n=10)$} \\
\hline F1 & $\begin{array}{l}\text { 1-O- } \beta \text {-D-glucopyranosyl } \\
\text {-paeonisuffrone }\end{array}$ & Capsule & $\mathrm{C} 16 \mathrm{H} 24 \mathrm{O} 9$ & 360.36 & NF (Figure 2) & LC-MS/UPLC-ESI-Q-TOF-MS [37] \\
\hline F2 & $\begin{array}{l}\text { 6-O- } \beta \text {-D-glucopyranosyl } \\
\text { lactinolide }\end{array}$ & Capsule & $\mathrm{C} 16 \mathrm{H} 26 \mathrm{O} 9$ & 362.37 & NF (Figure 2) & LC-MS/UPLC-ESI-Q-TOF-MS [37] \\
\hline F3 & Albiflorin R1 & Capsule & $\mathrm{C} 23 \mathrm{H} 28 \mathrm{O} 11$ & 480.466 & $\begin{array}{l}5317181 \text { (CID), } \\
50163461(\mathrm{SID})\end{array}$ & $\begin{array}{l}\text { LC-MS/UPLC-ESI-Q-TOF-MS [37], } \\
\text { chromatography on silica gel, Sephdex LH-20 } \\
\text { columns and prep-HPLC-NMR [53] }\end{array}$ \\
\hline F4 & Digallic acid & Capsule & C14H10O9 & 322.225 & 341 & LC-MS/UPLC-ESI-Q-TOF-MS [37] \\
\hline F5 & Ethyl gallate & Capsule & C9H10O5 & 198.174 & 13250 & $\begin{array}{l}\text { LC-MS/UPLC-ESI-Q-TOF-MS [37], multiple } \\
\text { chromatographic methods [42] }\end{array}$ \\
\hline F6 & Galloylsucrose/isomer & Capsule & C19H26O15 & 494.402 & 129629059 & LC-MS/UPLC-ESI-Q-TOF-MS [37] \\
\hline F7 & Isomaltopaeoniflorin sulfonate & Capsule & C29H38O18S & 706.66 & NF (Figure 2) & LC-MS/UPLC-ESI-Q-TOF-MS [37] \\
\hline F8 & Oxypaeoniflorin sulfonate & Capsule & $\mathrm{C} 23 \mathrm{H} 28 \mathrm{O} 14 \mathrm{~S}$ & 560.523 & 71455848 & LC-MS/UPLC-ESI-Q-TOF-MS [37] \\
\hline F9 & Paeoniflorin & Capsule, pill, tablet & $\mathrm{C} 23 \mathrm{H} 28 \mathrm{O} 11$ & 480.466 & 442534 & $\begin{array}{l}\text { LC-MS/UPLC-ESI-Q-TOF-MS [37], HPLC } \\
\text { [47,50,51,59,60], RP-HPLC [52], } \\
\text { chromatography on silica gel, Sephdex LH-20 } \\
\text { columns and prep-HPLC-NMR [53], } \\
\text { HPLC-ESI-QTOF/MS [43], DGLC [44], UPLC } \\
\text { [41], HPLC-MS/MS [45] }\end{array}$ \\
\hline F10 & Paeoniflorin sulfonate & Capsule & $\mathrm{C} 23 \mathrm{H} 28 \mathrm{O} 14 \mathrm{~S}$ & 560.523 & 101382399 & $\begin{array}{c}\text { LC-MS/UPLC-ESI-Q-TOF-MS [37], } \\
\text { HPLC-ESI-QTOF/MS [43] }\end{array}$ \\
\hline G & \multicolumn{6}{|c|}{ Poria $(n=30)$} \\
\hline G1 & $16 \alpha$-Hydroxytrametenolic acid & Capsule & $\mathrm{C} 30 \mathrm{H} 48 \mathrm{O} 4$ & 472.71 & 132285301 & $\begin{array}{l}\text { LC-MS/UPLC-ESI-Q-TOF-MS [37], } \\
\text { UPLC/Q-TOF-MS [61] }\end{array}$ \\
\hline G2 & $\begin{array}{l}16 \alpha \text {-Hydroxydehydrotrametenolic } \\
\text { acid }\end{array}$ & Capsule & $\mathrm{C} 30 \mathrm{H} 46 \mathrm{O} 4$ & 470.694 & 10743008 & UPLC/Q-TOF-MS [61] \\
\hline G3 & 2,3,6-Trimethylbenzoic acid & Capsule & $\mathrm{C} 10 \mathrm{H} 12 \mathrm{O} 2$ & 164.204 & 17314 & LC-MS/UPLC-ESI-Q-TOF-MS [37] \\
\hline G4 & 25-Hydroxypachymic acid & Capsule & $\mathrm{C} 33 \mathrm{H} 52 \mathrm{O} 6$ & 544.76 & NF (Figure 2) & LC-MS/UPLC-ESI-Q-TOF-MS [37] \\
\hline G5 & $\begin{array}{c}\text { 31-Hydroxyl-16-O-acetylpachymic } \\
\text { acid }\end{array}$ & Capsule & $\mathrm{C} 38 \mathrm{H} 52 \mathrm{O} 5$ & 588.81 & NF (Figure 2) & LC-MS/UPLC-ESI-Q-TOF-MS [37] \\
\hline
\end{tabular}


Table 2. Cont

\begin{tabular}{|c|c|c|c|c|c|c|}
\hline ID & List of Chemical Compounds & Form of GFW & Molecular Formula & Molecular Weight (g/mol) & PubChem CID/SID & Methods \\
\hline G6 & 3-epidehydropachymic acid & Capsule & C33H50O5 & 526.758 & 15226716 & $\begin{array}{c}\text { Chromatography on silica gel, Sephdex LH-20 } \\
\text { columns, D-101 macroporous resin column, } \\
\text { and reverse RP-18 column and } \\
\text { HPLC-NMR/MS [55], HPLC [62], } \\
\text { UPLC/Q-TOF-MS [61] }\end{array}$ \\
\hline G7 & 3-Epidehydrotumulosic acid & Capsule & $\mathrm{C} 31 \mathrm{H} 48 \mathrm{O} 4$ & 484.721 & 10005581 & $\begin{array}{l}\text { LC-MS/UPLC-ESI-Q-TOF-MS [37], } \\
\text { chromatography on silica gel, Sephdex LH-20 } \\
\text { columns, D-101 macroporous resin column, } \\
\text { and reverse RP-18 column and } \\
\text { HPLC-NMR/MS [55], UPLC/Q-TOF-MS [61] }\end{array}$ \\
\hline G8 & $\begin{array}{c}\text { 3-O-acetyl-16 } \alpha \\
\text { hydroxytrametenolic acid }\end{array}$ & Capsule & $\mathrm{C} 32 \mathrm{H} 50 \mathrm{O} 5$ & 514.747 & 9958136 & $\begin{array}{l}\text { LC-MS/UPLC-ESI-Q-TOF-MS [37], } \\
\text { UPLC/Q-TOF-MS [61] }\end{array}$ \\
\hline G9 & $\begin{array}{l}\text { 3-O-acetyl-16 } \alpha \text {-hydroxy } \\
\text { dehydrotrametenolic acid }\end{array}$ & Capsule & $\mathrm{C} 32 \mathrm{H} 48 \mathrm{O} 5$ & 512.731 & 15226714 & $\begin{array}{l}\text { LC-MS/UPLC-ESI-Q-TOF-MS [37], } \\
\text { UPLC/Q-TOF-MS [61] }\end{array}$ \\
\hline G10 & $\begin{array}{l}\text { 3-oxo-6,16 } \alpha \text {-dihydroxy-lanosta } \\
-7,9(11), 24(31) \text {-trien-21-oic acid }\end{array}$ & Capsule & $\mathrm{C} 30 \mathrm{H} 44 \mathrm{O} 5$ & 484.67 & $\begin{array}{l}\text { NF (Figure 2) } \\
\text { (structure found in } \\
\text { [63]) }\end{array}$ & UPLC/Q-TOF-MS [61] \\
\hline G11 & $\begin{array}{c}\text { 3-oxo-6,16 } \alpha \text {-dihydroxy } \\
\text {-lanosta-8,24-diene-21-oic acid } \\
3 \beta, 16 \alpha \text {-Dihydroxy }\end{array}$ & Capsule & $\mathrm{NF}$ & NF & $\begin{array}{l}\text { NF (structure not } \\
\text { available) }\end{array}$ & UPLC/Q-TOF-MS [61] \\
\hline G12 & $\begin{array}{c}\text {-lanosta-7,9(11),24-trien-21-oic } \\
\text { acid }\end{array}$ & Capsule & $\mathrm{C} 30 \mathrm{H} 46 \mathrm{O} 4$ & 470.68 & NF (Figure 2) & LC-MS/UPLC-ESI-Q-TOF-MS [37] \\
\hline G13 & $\begin{array}{l}3 \beta-\mathrm{O}-\mathrm{p}-\mathrm{Hydroxybenzoyl} \text {-dehydro } \\
\text { tumulosic acid }\end{array}$ & Capsule & $\mathrm{C} 38 \mathrm{H} 52 \mathrm{O} 6$ & 604.828 & 5318155 & LC-MS/UPLC-ESI-Q-TOF-MS [37] \\
\hline G14 & $\begin{array}{c}6 \alpha \text {-Hydroxydehydropachymic } \\
\text { acid }\end{array}$ & Capsule & $\mathrm{C} 33 \mathrm{H} 50 \mathrm{O} 6$ & 542.74 & NF (Figure 2) & LC-MS/UPLC-ESI-Q-TOF-MS [37] \\
\hline G15 & Dehydroeburicoic acid & Capsule & $\mathrm{C} 31 \mathrm{H} 48 \mathrm{O} 3$ & 468.722 & 15250826 & $\begin{array}{c}\text { LC-MS/UPLC-ESI-Q-TOF-MS [37], } \\
\text { UPLC-MS/MS [64], UPLC/Q-TOF-MS [61] } \\
\text { LC-MS/UPLC-ESI-Q-TOF-MS [37], }\end{array}$ \\
\hline G16 & Dehydropachymic acid & Capsule & С33H50O5 & 526.758 & 15226717 & $\begin{array}{l}\text { UPLC-MS/MS [64], HPLC [62], } \\
\text { UPLC/Q-TOF-MS [61] }\end{array}$ \\
\hline G17 & Dehydrotrametenolic acid & Capsule & $\mathrm{C} 32 \mathrm{H} 48 \mathrm{O} 3$ & 480.733 & 129539661 & $\begin{array}{l}\text { LC-MS/UPLC-ESI-Q-TOF-MS [37], } \\
\text { UPLC-MS/MS [64], UPLC/Q-TOF-MS [61] }\end{array}$ \\
\hline G18 & Dehydrotumulosic acid & Capsule & $\mathrm{C} 31 \mathrm{H} 48 \mathrm{O} 4$ & 484.721 & 15225964 & $\begin{array}{l}\text { LC-MS/UPLC-ESI-Q-TOF-MS [37], } \\
\text { UPLC-MS/MS [64], chromatography on silica } \\
\text { gel, Sephdex LH-20 columns, D-101 } \\
\text { macroporous resin column, and reverse RP-18 } \\
\text { column and HPLC-NMR/MS [55], HPLC [62], } \\
\text { UPLC/Q-TOF-MS [61] }\end{array}$ \\
\hline
\end{tabular}


Table 2. Cont

\begin{tabular}{|c|c|c|c|c|c|c|}
\hline ID & List of Chemical Compounds & Form of GFW & Molecular Formula & Molecular Weight (g/mol) & PubChem CID/SID & Methods \\
\hline G19 & Eburicoic acid & Capsule & C31H50O3 & 470.738 & 73402 & $\begin{array}{l}\text { LC-MS/UPLC-ESI-Q-TOF-MS [37], } \\
\text { UPLC/Q-TOF-MS [61] }\end{array}$ \\
\hline G20 & Pachymic acid & Capsule & C33H52O5 & 528.774 & 5484385 & $\begin{array}{l}\text { LC-MS/UPLC-ESI-Q-TOF-MS [37], } \\
\text { UPLC-MS/MS [64], HPLC-MS/MS [45], } \\
\text { UPLC/Q-TOF-MS [61] }\end{array}$ \\
\hline G21 & Pachymic acid methyl ester & Capsule & С $34 \mathrm{H} 54 \mathrm{O} 5$ & 542.79 & NF (Figure 2) & LC-MS/UPLC-ESI-Q-TOF-MS [37] \\
\hline G22 & Polyporenic acid C & Capsule & $\mathrm{C} 31 \mathrm{H} 46 \mathrm{O} 4$ & 482.705 & 9805290 & $\begin{array}{l}\text { LC-MS/UPLC-ESI-Q-TOF-MS [37], } \\
\text { UPLC-MS/MS [64], chromatography on silica } \\
\text { gel, Sephdex LH-20 columns, D-101 } \\
\text { macroporous resin column, and reverse RP-18 } \\
\text { column and HPLC-NMR/MS [55], HPLC [62], } \\
\text { UPLC/Q-TOF-MS [61] }\end{array}$ \\
\hline G23 & Poricoic acid A & Capsule & $\mathrm{C} 31 \mathrm{H} 46 \mathrm{O} 5$ & 498.704 & 5471851 & LC-MS/UPLC-ESI-Q-TOF-MS [37] \\
\hline G24 & Poricoic acid AE & Capsule & C33H50O5 & 526.758 & 102480392 & LC-MS/UPLC-ESI-Q-TOF-MS [37] \\
\hline G25 & Poricoic acid AM & Capsule & $\mathrm{C} 32 \mathrm{H} 48 \mathrm{O} 5$ & 512.731 & 46882717 & LC-MS/UPLC-ESI-Q-TOF-MS [37] \\
\hline G26 & Poricoic acid B & Capsule & $\mathrm{C} 30 \mathrm{H} 44 \mathrm{O} 5$ & 484.677 & 5471852 & LC-MS/UPLC-ESI-Q-TOF-MS [37] \\
\hline G27 & Poricoic acid BM & Capsule & C31H46O5 & 498.704 & 15225967 & LC-MS/UPLC-ESI-Q-TOF-MS [37] \\
\hline G28 & Poricoic acid C & Capsule & $\mathrm{C} 31 \mathrm{H} 46 \mathrm{O} 4$ & 482.705 & 16757534 & LC-MS/UPLC-ESI-Q-TOF-MS [37] \\
\hline G29 & Poricoic acid DM & Capsule & $\mathrm{C} 32 \mathrm{H} 48 \mathrm{O} 6$ & 528.73 & 44424830 & LC-MS/UPLC-ESI-Q-TOF-MS [37] \\
\hline G30 & Tumulosic acid & Capsule & C31H50O4 & 486.737 & 12314446 & $\begin{array}{l}\text { LC-MS/UPLC-ESI-Q-TOF-MS [37], } \\
\text { UPLC/Q-TOF-MS [61] }\end{array}$ \\
\hline $\mathbf{H}$ & \multicolumn{6}{|c|}{ Unclarified $(n=44)$} \\
\hline H1 & $\begin{array}{l}\text { (2R)-[(6-O- } \beta \text {-D-glucopyranosyl- } \beta \\
\text {-D-glucopyranosyl) oxy] } \\
\text { (phenyl) ethanoic acid }\end{array}$ & Capsule & $\mathrm{C} 20 \mathrm{H} 28 \mathrm{O} 13$ & 475. 145 & NF (Figure 2) & HPLC-ESI-Q-TOF/MS [43] \\
\hline $\mathrm{H} 2$ & 2,5-dihydroxy-4-methylacetophenone & Capsule & $\mathrm{C} 9 \mathrm{H} 10 \mathrm{O} 3$ & 166.174 & NF (Figure 2) & Multiple chromatographic methods [42] \\
\hline H3 & 4-o-methylbenzoylpaeoniflorin & Capsule & С $31 \mathrm{H} 34 \mathrm{O} 12$ & 598.601 & 46883517 & $\begin{array}{l}\text { Chromatography on silica gel, Sephdex LH-20 } \\
\text { columns and prep-HPLC-NMR [53] }\end{array}$ \\
\hline $\mathrm{H} 4$ & 4-o-methylgalloylpaeoniflorin & Capsule & С31H34O15 & 646.598 & 46883518 & $\begin{array}{l}\text { Chromatography on silica gel, Sephdex LH-20 } \\
\text { columns and prep-HPLC-NMR [53] }\end{array}$ \\
\hline H5 & Adenosine & Capsule & $\mathrm{C} 10 \mathrm{H} 13 \mathrm{~N} 5 \mathrm{O} 4$ & 267.245 & 60961 & $\begin{array}{c}\text { Chromatography on silica gel, Sephdex LH-20 } \\
\text { columns, D-101 macroporous resin column, } \\
\text { and reverse RP-18 column and } \\
\text { HPLC-NMR/MS [55] }\end{array}$ \\
\hline
\end{tabular}


Table 2. Cont

\begin{tabular}{|c|c|c|c|c|c|c|}
\hline ID & List of Chemical Compounds & Form of GFW & Molecular Formula & Molecular Weight (g/mol) & PubChem CID/SID & Methods \\
\hline H6 & Affinoside & Capsule & $\mathrm{C} 20 \mathrm{H} 28 \mathrm{O} 12$ & 460.432 & 11972427 & Multiple chromatographic methods [42] \\
\hline $\mathrm{H} 7$ & Alanine & Capsule & $\mathrm{C} 3 \mathrm{H} 7 \mathrm{NO} 2$ & 89.094 & 5950 & $\begin{array}{c}\text { Chromatography on silica gel, Sephdex LH-20 } \\
\text { columns, D-101 macroporous resin column, } \\
\text { and reverse RP-18 column and } \\
\text { HPLC-NMR/MS [55] }\end{array}$ \\
\hline H8 & A-Amyrin acetate & Capsule & $\mathrm{C} 32 \mathrm{H} 52 \mathrm{O} 2$ & 468.766 & 92842 & $\begin{array}{l}\text { Chromatography on silica gel and } \\
\text { polydextran gel columns and } \\
\text { prep-HPLC-NMR/MS [54] }\end{array}$ \\
\hline H9 & A-D-Glucose & Capsule & C6H12O6 & 180.156 & 79025 & $\begin{array}{c}\text { Chromatography on silica gel, Sephdex LH-20 } \\
\text { columns, D-101 macroporous resin column, } \\
\text { and reverse RP-18 column and } \\
\text { HPLC-NMR/MS [56] }\end{array}$ \\
\hline $\mathrm{H} 10$ & Apigenin & Capsule & C15H10O5 & 270.24 & 5280443 & $\begin{array}{l}\text { Chromatography on silica gel and } \\
\text { polydextran gel columns and } \\
\text { prep-HPLC-NMR/MS [54] }\end{array}$ \\
\hline H11 & Arginine & Capsule & $\mathrm{C} 6 \mathrm{H} 14 \mathrm{~N} 4 \mathrm{O} 2$ & 174.204 & 6322 & $\begin{array}{c}\text { Chromatography on silica gel, Sephdex LH-20 } \\
\text { columns, D-101 macroporous resin column, } \\
\text { and reverse RP-18 column and } \\
\text { HPLC-NMR/MS [56] }\end{array}$ \\
\hline H12 & Astragalin & Capsule & $\mathrm{C} 21 \mathrm{H} 20 \mathrm{O} 11$ & 448.38 & 5282102 & $\begin{array}{l}\text { Chromatography on silica gel and } \\
\text { polydextran gel columns and } \\
\text { prep-HPLC-NMR/MS [54] }\end{array}$ \\
\hline H13 & Benzoic acid & Capsule & C7H6O2 & 122.123 & 243 & HPLC-ESI-QTOF/MS [43], UPLC [41] \\
\hline H14 & Benzoylpaeoniflorin & Capsule & $\mathrm{C} 30 \mathrm{H} 32 \mathrm{O} 12$ & 584.574 & 21631106 & HPLC-ESI-QTOF/MS [43], UPLC [41] \\
\hline H15 & $\begin{array}{l}\text { Benzyl- } \beta \text {-D-glucopyranosyl } \\
-(1 \rightarrow 6)-\beta \text {-D-glucopyranoside }\end{array}$ & Capsule & $\mathrm{C} 18 \mathrm{H} 26 \mathrm{O} 10$ & 402.39 & NF (Figure 2) & Multiple chromatographic methods [42] \\
\hline H16 & B-Amyrin acetate & Capsule & $\mathrm{C} 32 \mathrm{H} 52 \mathrm{O} 2$ & 468.766 & 92156 & $\begin{array}{c}\text { Chromatography on silica gel and } \\
\text { polydextran gel columns and } \\
\text { prep-HPLC-NMR/MS [54] }\end{array}$ \\
\hline H17 & Caffeic acid & Capsule & $\mathrm{C} 9 \mathrm{H} 8 \mathrm{O} 4$ & 180.159 & 689043 & $\begin{array}{c}\text { Chromatography on silica gel, Sephdex LH-20 } \\
\text { columns, D-101 macroporous resin column, } \\
\text { and reverse RP-18 column and } \\
\text { HPLC-NMR/MS [55] }\end{array}$ \\
\hline $\mathrm{H} 18$ & Campesterol & Capsule & $\mathrm{C} 28 \mathrm{H} 48 \mathrm{O}$ & 400.691 & 173183 & $\begin{array}{l}\text { Chromatography on silica gel and } \\
\text { polydextran gel columns and } \\
\text { prep-HPLC-NMR/MS [54] }\end{array}$ \\
\hline
\end{tabular}


Table 2. Cont

\begin{tabular}{|c|c|c|c|c|c|c|}
\hline ID & List of Chemical Compounds & Form of GFW & Molecular Formula & Molecular Weight (g/mol) & PubChem CID/SID & Methods \\
\hline H19 & Catechin & Capsule & $\mathrm{C} 15 \mathrm{H} 14 \mathrm{O} 6$ & 290.271 & 9064 & $\begin{array}{l}\text { Chromatography on silica gel and } \\
\text { polydextran gel columns and } \\
\text { prep-HPLC-NMR/MS [54] }\end{array}$ \\
\hline $\mathrm{H} 20$ & Cinnamyl alcohol & Capsule & $\mathrm{C} 9 \mathrm{H} 10 \mathrm{O}$ & 134.178 & 5315892 & $\begin{array}{l}\text { Chromatography on silica gel and } \\
\text { polydextran gel columns and } \\
\text { prep-HPLC-NMR/MS [54] }\end{array}$ \\
\hline $\mathrm{H} 21$ & Coumarin & Capsule & $\mathrm{C} 9 \mathrm{H} 6 \mathrm{O} 2$ & 146.145 & 323 & $\begin{array}{l}\text { Chromatography on silica gel and } \\
\text { polydextran gel columns and } \\
\text { prep-HPLC-NMR/MS [54] }\end{array}$ \\
\hline $\mathrm{H} 22$ & Epicatechin & Capsule & $\mathrm{C} 15 \mathrm{H} 14 \mathrm{O} 6$ & 290.271 & 72276 & $\begin{array}{l}\text { Chromatography on silica gel and } \\
\text { polydextran gel columns and } \\
\text { prep-HPLC-NMR/MS [54] }\end{array}$ \\
\hline $\mathrm{H} 23$ & $\begin{array}{l}\text { Ergosta-4, 6, } 8(14), \\
\text { 22-tetraen-3-one }\end{array}$ & Capsule & $\mathrm{C} 28 \mathrm{H} 40 \mathrm{O}$ & 392.627 & 6441416 & $\begin{array}{l}\text { Chromatography on silica gel and } \\
\text { polydextran gel columns and } \\
\text { prep-HPLC-NMR/MS [54] }\end{array}$ \\
\hline $\mathrm{H} 24$ & Ergosterol & Capsule & $\mathrm{C} 28 \mathrm{H} 44 \mathrm{O}$ & 396.659 & 444679 & $\begin{array}{l}\text { Chromatography on silica gel and } \\
\text { polydextran gel columns and } \\
\text { prep-HPLC-NMR/MS [54] }\end{array}$ \\
\hline $\mathrm{H} 25$ & Galactitol & Capsule & C6H14O6 & 182.172 & 11850 & $\begin{array}{c}\text { Chromatography on silica gel, Sephdex LH-20 } \\
\text { columns, D-101 macroporous resin column, } \\
\text { and reverse RP-18 column and } \\
\text { HPLC-NMR/MS [55] }\end{array}$ \\
\hline $\mathrm{H} 26$ & Guanosine & Capsule & $\mathrm{C} 10 \mathrm{H} 13 \mathrm{~N} 5 \mathrm{O} 5$ & 283.244 & 6802 & $\begin{array}{c}\text { Chromatography on silica gel, Sephdex LH-20 } \\
\text { columns, D-101 macroporous resin column, } \\
\text { and reverse RP-18 column and } \\
\text { HPLC-NMR/MS [55] }\end{array}$ \\
\hline $\mathrm{H} 27$ & Heneicosanoic acid & Capsule & $\mathrm{C} 21 \mathrm{H} 42 \mathrm{O} 2$ & 326.565 & 16898 & $\begin{array}{c}\text { Chromatography on silica gel and } \\
\text { polydextran gel columns and } \\
\text { prep-HPLC-NMR/MS [54] }\end{array}$ \\
\hline $\mathrm{H} 28$ & Hyperoside & Capsule & $\mathrm{C} 21 \mathrm{H} 20 \mathrm{O} 12$ & 464.379 & 5281643 & $\begin{array}{l}\text { Chromatography on silica gel and } \\
\text { polydextran gel columns and } \\
\text { prep-HPLC-NMR/MS [54] }\end{array}$ \\
\hline H29 & Isomaltopaeoniflorin & Capsule & $\mathrm{C} 29 \mathrm{H} 38 \mathrm{O} 16$ & 642.607 & 101001429 & $\begin{array}{l}\text { Chromatography on silica gel, Sephdex LH-20 } \\
\text { columns and prep-HPLC-NMR [53] }\end{array}$ \\
\hline H30 & Kaemferol & Capsule & C15H10O6 & 286.239 & 5280863 & $\begin{array}{l}\text { Chromatography on silica gel and } \\
\text { polydextran gel columns and } \\
\text { prep-HPLC-NMR/MS [54] }\end{array}$ \\
\hline
\end{tabular}


Table 2. Cont.

\begin{tabular}{|c|c|c|c|c|c|c|}
\hline ID & List of Chemical Compounds & Form of GFW & Molecular Formula & Molecular Weight (g/mol) & PubChem CID/SID & Methods \\
\hline H31 & Leucine & Capsule & $\mathrm{C} 6 \mathrm{H} 13 \mathrm{NO} 2$ & 131.175 & 6106 & $\begin{array}{c}\text { Chromatography on silica gel, Sephdex LH-20 } \\
\text { columns, D-101 macroporous resin column, } \\
\text { and reverse RP-18 column and } \\
\text { HPLC-NMR/MS [55] }\end{array}$ \\
\hline H32 & $\begin{array}{l}\text { O- } \beta \text {-D-Gentiobiosyl- } \\
\text { D-(-)-mandelamide }\end{array}$ & Capsule & $\mathrm{C} 20 \mathrm{H} 29 \mathrm{NO} 12$ & 498. 158 & 181802 & HPLC-ESI-QTOF/MS [43] \\
\hline H33 & Paeonidanin A & Capsule & C31H34O12 & 598.601 & 44253993 & $\begin{array}{l}\text { Chromatography on silica gel, Sephdex LH-20 } \\
\text { columns and prep-HPLC-NMR [53] }\end{array}$ \\
\hline H34 & Paeonidanin B & Capsule & С $31 \mathrm{H} 34 \mathrm{O} 15$ & 646.598 & 102417825 & $\begin{array}{l}\text { Chromatography on silica gel, Sephdex LH-20 } \\
\text { columns and prep-HPLC-NMR [53] }\end{array}$ \\
\hline H35 & Paeoniflorin B & Capsule & $\mathrm{C} 36 \mathrm{H} 42 \mathrm{O} 17$ & 746.715 & 71452334 & $\begin{array}{l}\text { Chromatography on silica gel, Sephdex LH-20 } \\
\text { columns and prep-HPLC-NMR [53] }\end{array}$ \\
\hline H36 & P-Coumaric acid & Capsule & $\mathrm{C} 9 \mathrm{H} 8 \mathrm{O} 3$ & 164.16 & 637542 & Multiple chromatographic methods [42] \\
\hline H37 & Proline & Capsule & $\mathrm{C} 5 \mathrm{H} 9 \mathrm{NO} 2$ & 115.132 & 145742 & $\begin{array}{c}\text { Chromatography on silica gel, Sephdex LH-20 } \\
\text { columns, D-101 macroporous resin column, } \\
\text { and reverse RP-18 column and } \\
\text { HPLC-NMR/MS [55] }\end{array}$ \\
\hline H38 & Quercetin & Capsule & $\mathrm{C} 15 \mathrm{H} 10 \mathrm{O} 7$ & 302.238 & 5280343 & $\begin{array}{l}\text { Chromatography on silica gel and } \\
\text { polydextran gel columns and } \\
\text { prep-HPLC-NMR/MS [54] }\end{array}$ \\
\hline H39 & Syringaresinol & Capsule & $\mathrm{C} 22 \mathrm{H} 26 \mathrm{O} 8$ & 418.442 & 443023 & $\begin{array}{l}\text { Chromatography on silica gel and } \\
\text { polydextran gel columns and } \\
\text { prep-HPLC-NMR/MS [54] }\end{array}$ \\
\hline $\mathrm{H} 40$ & Syringic acid & Capsule & $\mathrm{C} 9 \mathrm{H} 10 \mathrm{O} 5$ & 198.174 & 10742 & $\begin{array}{l}\text { Chromatography on silica gel and } \\
\text { polydextran gel columns and } \\
\text { prep-HPLC-NMR/MS [54], } \\
\text { HPLC-ESI-QTOF/MS [43] }\end{array}$ \\
\hline $\mathrm{H} 41$ & Trans-2-Methoxycinnamic acid & Capsule & $\mathrm{C} 10 \mathrm{H} 10 \mathrm{O} 3$ & 178.187 & 734154 & Multiple chromatographic methods [42] \\
\hline $\mathrm{H} 42$ & Trehalose & Capsule & $\mathrm{C} 12 \mathrm{H} 22 \mathrm{O} 11$ & 342.297 & 7427 & $\begin{array}{c}\text { Chromatography on silica gel, Sephdex LH-20 } \\
\text { columns, D-101 macroporous resin column, } \\
\text { and reverse RP-18 column and } \\
\text { HPLC-NMR/MS [55] }\end{array}$ \\
\hline $\mathrm{H} 43$ & Umbelliferone & Capsule & $\mathrm{C} 9 \mathrm{H} 6 \mathrm{O} 3$ & 162.144 & 5281426 & $\begin{array}{l}\text { Chromatography on silica gel and } \\
\text { polydextran gel columns and } \\
\text { prep-HPLC-NMR/MS [54] }\end{array}$ \\
\hline $\mathrm{H} 44$ & Vanillic acid & Capsule & $\mathrm{C} 8 \mathrm{H} 8 \mathrm{O} 4$ & 168.148 & 8468 & Multiple chromatographic methods [42] \\
\hline
\end{tabular}


Table 2. Cont.

\begin{tabular}{|c|c|c|c|c|c|c|}
\hline ID & List of Chemical Compounds & Form of GFW & Molecular Formula & Molecular Weight (g/mol) & PubChem CID/SID & Methods \\
\hline $\mathbf{J}$ & \multicolumn{6}{|c|}{ Inorganic Elements $(n=29)$} \\
\hline J1 & Aluminum & Capsule & $\mathrm{Al}$ & 26.982 & 5359268 & ICP-MS [65] \\
\hline $\mathrm{J} 2$ & Arsenic & Capsule & As & 74.922 & 5359596 & ICP-MS [65] \\
\hline $\mathrm{J} 3$ & Boron & Capsule & B & 10.81 & 5462311 & ICP-MS [65] \\
\hline $\mathrm{J} 4$ & Barium & Capsule & $\mathrm{Ba}$ & 137.327 & 5355457 & ICP-MS [65] \\
\hline $\mathrm{J} 5$ & Beryllium & Capsule & Be & 9.012 & 5460467 & ICP-MS [65] \\
\hline J6 & Bismuth & Capsule & $\mathrm{Bi}$ & 208.98 & 5359367 & ICP-MS [65] \\
\hline $\mathrm{J} 7$ & Calcium & Capsule & $\mathrm{Ca}$ & 40.078 & 5460341 & ICP-MS [65] \\
\hline $\mathrm{J} 8$ & Cadmium & Capsule & $\mathrm{Cd}$ & 112.414 & 23973 & ICP-MS [65] \\
\hline J9 & Cobalt & Capsule & Co & 58.933 & 104730 & ICP-MS [65] \\
\hline J10 & Chromium & Capsule & $\mathrm{Cr}$ & 51.996 & 23976 & ICP-MS [65] \\
\hline $\mathrm{J} 11$ & Copper & Capsule & $\mathrm{Cu}$ & 63.546 & 23978 & ICP-MS [65] \\
\hline J12 & Iron & Capsule & $\mathrm{Fe}$ & 55.845 & 23925 & ICP-MS [65] \\
\hline $\mathrm{J} 13$ & Gallium & Capsule & $\mathrm{Ga}$ & 69.723 & 5360835 & ICP-MS [65] \\
\hline J14 & Mercury & Capsule & $\mathrm{Hg}$ & 200.592 & 23931 & ICP-MS [65] \\
\hline J15 & Lithium & Capsule & $\mathrm{Li}$ & 6.94 & 3028194 & ICP-MS [65] \\
\hline J16 & Magnesium & Capsule & $\mathrm{Mg}$ & 24.305 & 5462224 & ICP-MS [65] \\
\hline J17 & Manganese & Capsule & $\mathrm{Mn}$ & 54.938 & 23930 & ICP-MS [65] \\
\hline $\mathrm{J} 18$ & Molybdenum & Capsule & Mo & 95.95 & 23932 & ICP-MS [65] \\
\hline J19 & Sodium & Capsule & $\mathrm{Na}$ & 22.99 & 5360545 & ICP-MS [65] \\
\hline $\mathrm{J} 20$ & Nickel & Capsule & $\mathrm{Ni}$ & 58.693 & 935 & ICP-MS [65] \\
\hline $\mathrm{J} 21$ & Lead & Capsule & $\mathrm{Pb}$ & 207.2 & 5352425 & ICP-MS [65] \\
\hline $\mathrm{J} 22$ & Antimony & Capsule & $\mathrm{Sb}$ & 121.76 & 5354495 & ICP-MS [65] \\
\hline $\mathrm{J} 23$ & Selenium & Capsule & Se & 78.971 & 6326970 & ICP-MS [65] \\
\hline $\mathrm{J} 24$ & Tin & Capsule & Sn & 118.71 & 5352426 & ICP-MS [65] \\
\hline $\mathrm{J} 25$ & Strontium & Capsule & $\mathrm{Sr}$ & 87.62 & 5359327 & ICP-MS [65] \\
\hline $\mathrm{J} 26$ & Titanium & Capsule & $\mathrm{Ti}$ & 47.867 & 23963 & ICP-MS [65] \\
\hline $\mathrm{J} 27$ & Thallium & Capsule & $\mathrm{Tl}$ & 204.38 & 5359464 & ICP-MS [65] \\
\hline $\mathrm{J} 28$ & Vanadium & Capsule & $\mathrm{V}$ & 50.941 & 23990 & ICP-MS [65] \\
\hline $\mathrm{J} 29$ & Zinc & Capsule & $\mathrm{Zn}$ & 65.38 & 23994 & ICP-MS [65] \\
\hline
\end{tabular}

Notes: CID: compound identification number; DGLC: dual gradient liquid chromatography; ESI: electrospray ionization; ICP: inductively coupled plasma; LC: liquid chromatography; MS:
mass spectrometry; NF: Not found; NMR: nuclear magnetic resonance spectroscopy; Q: quadrupole; RP: reverse phase; SID: substance identification number; SPE: solid-phase extraction; TOF: time of flight; UPLC: ultra performance liquid chromatography. Corresponding molecular structures refer to PubChem and Figure 2. 


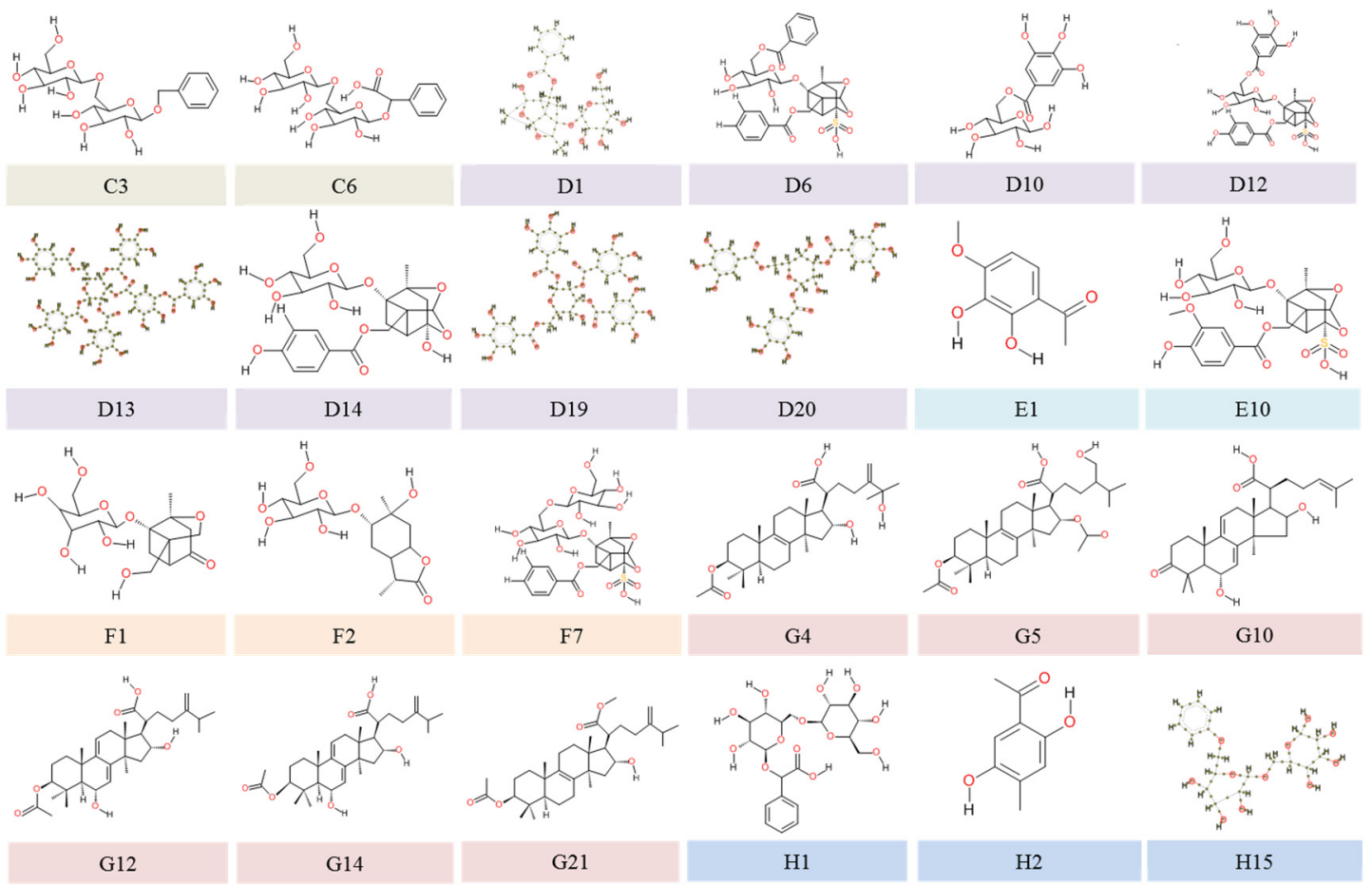

Figure 2. Molecular structures of the 24 chemical constituents of Guizhi Fuling Wan. Note: C3: Benzol- $\beta$-gentiobioside, C6: Mandelic acid gentiobioside/isomer, D1: 4'-O-galloypaeoniflorin, D6: Benzoylpaeoniflorin sulfate, D10: Galloyglucose/isomer, D12: Galloylpaeoniflorin sulfonate, D13: Hexagalloyl glucose, D14: Isoxypaeoniflora, D19: Tetragalloy glucose, D20: Trigalloy glucose, E1: 3-hydroxypaeonol, E10: Mudanpioside E sulfonate, F1: 1-O- $\beta$-D-glucopyranosyl-paeonisuffrone, F2: 6-O- $\beta$-D-glucopyranosyl lactinolide, F7: Isomaltopaeoniflorin sulfonate, G4: 25-Hydroxypachymic acid, G5: 31-Hydroxyl-16-O-acetylpachymic acid, G10: 3-oxo-6,16 $\alpha$-dihydroxy-lanosta-7,9(11),24(31) -trien-21-oic acid, G12: 3 $\beta, 16 \alpha$-Dihydroxy-lanosta-7,9(11),24-trien-21-oic acid, G14: $6 \alpha$-Hydroxyd ehydropachymic acid, G21: Pachymic acid methyl ester, H1: (2R)-[(6-O- $\beta$-D-glucopyranosyl - $\beta$-D-glucopyranosyl) oxy] (phenyl) ethanoic acid, H2: 2,5-dihydroxy-4-methylacetophenone, H15: Benzyl- $\beta$-D-glucopyranosyl-( $1 \rightarrow 6)$ - $\beta$-D-glucopyranoside.

\subsection{Pharmacodynamic Effects of GFW on MHFs}

Characteristics and results of included in vivo studies are summarized in Table 3. The results from the quality assessment of included in vivo experimental studies are presented in Figure 3. The results suggested that GFW had a similar action to $E_{2}$ on managing skin temperature by restoring the plasma level of CGRP: it increased the ovariectomy-induced CGRP reduction $[66,67]$ and reduced CGRP-induced elevation of skin temperature in GnRH (gonadotropin-releasing hormone) analog-treated rats $[67,68]$. A dose-dependent skin temperature change was detected, showing that a significant inhibition effect on elevated skin temperature was observed at a dose of $1000 \mathrm{mg} / \mathrm{kg}[67,68]$. However, GFW had no significant effects on CGRP concentrations and CGRP mRNA levels in the dorsal root ganglia [66], which suggested minor effects on CGRP synthesis in an ovariectomized rat model. GFW had no significant effects on CGRP concentrations in the spinal cord [66], which suggested that it may have an inconsequential influence on CGRP's effects on the central nervous system in the ovariectomized rat model. 
Table 3. Characteristics of in vivo studies.

\begin{tabular}{|c|c|c|c|c|c|c|c|c|c|c|}
\hline Study ID & $\begin{array}{l}\text { Language; } \\
\text { Location }\end{array}$ & Model & Inducer & Age (week) & Weight $(\mathrm{g})$ & Experiments & Sample Size & Interventions (Daily Dose, Administration) & $\begin{array}{c}\text { Duration } \\
\text { (days) }\end{array}$ & $\begin{array}{l}\text { Included Outcome Measure } \\
\text { and Results }\end{array}$ \\
\hline \multirow[t]{3}{*}{$\begin{array}{l}\text { Noguchi et al., } \\
2003 \text { a [66] }\end{array}$} & English, Japan & $\begin{array}{l}\text { Sprague-Dawley } \\
\text { rats }\end{array}$ & $\begin{array}{l}\text { OVX, } \\
\text { capsaicin (1 } \\
\text { mg/kg, i.p.) } \\
\text { injection }\end{array}$ & 10 & $200-250$ & 1 & $32(8 / 8 / 8 / 8)$ & $\begin{array}{c}\text { 1. } \mathrm{E}_{2}(0.010 \mathrm{mg} / \mathrm{kg} \text {, s.c.); } \\
\text { 2. GFW }(1000 \mathrm{mg} / \mathrm{kg} \text {, p.o.); } \\
\text { 3. Distilled water }(10 \mathrm{~mL} / \mathrm{kg}, \text { p.o.) to OVX rats; } \\
\text { 4. Distilled water }(10 \mathrm{~mL} / \mathrm{kg} \text {, p.o.) to }\end{array}$ & 7 & $\begin{array}{l}\text { CGRP concentrations in } \\
\text { plasma } \uparrow \text { (restore) }\end{array}$ \\
\hline & & & ovx & & & 2 & $27(7 / 7 / 7 / 7)$ & sham-operated rats. & & $\begin{array}{l}\text { CGRP concentrations in the } \\
\text { spinal cord and dorsal root } \\
\text { ganglia (no sig) }\end{array}$ \\
\hline & & & & & & 3 & $20(5 / 5 / 5 / 5)$ & & & $\begin{array}{l}\text { CGRP mRNA levels in dorsal } \\
\text { root ganglia (no sig) }\end{array}$ \\
\hline \multirow[t]{5}{*}{$\begin{array}{l}\text { Noguchi et al., } \\
2003 \text { b [67] }\end{array}$} & English, Japan & $\begin{array}{l}\text { Sprague-Dawley } \\
\text { rats }\end{array}$ & ovx & 10 & $200-250$ & 4 & NS & $\begin{array}{l}\text { 1. GFW (100, } 300, \text { or } 1000 \mathrm{mg} / 10 \mathrm{~mL} \text { per kg, p.o., } n \\
=7 \text { or } 8 \text { i each group); } \\
\text { 2. } \mathrm{E}_{2}(0.010 \mathrm{mg} / \mathrm{mL} \text { per kg, s.c, } n=7) ; \\
\text { 3. Distilled water }(10 \mathrm{mg} / \mathrm{kg}, \text { p.o., } n=8) \text { to OVX } \\
\text { rats; } \\
\text { 4. GFW (1000 mg/10 mL per kg, p.o., } n=8) \text { to } \\
\text { sham-operated rats. }\end{array}$ & 7 & $\begin{array}{l}\text { CGRP-induced elevation of } \\
\text { skin temperature } \downarrow\end{array}$ \\
\hline & & & & & & 5 & $\begin{array}{c}45 \\
(11 / 13 / 12 / 9)\end{array}$ & $\begin{array}{l}\text { 1. GFW (1000 mg/10 mL per kg, p.o.); } \\
\text { 2. } \mathrm{E}_{2}(0.010 \mathrm{mg} / \mathrm{mL} \text { per kg, s.c.); } \\
\text { 3. Distilled water }(10 \mathrm{~mL} / \mathrm{kg} \text {, p.o.) to OVX rats; } \\
\text { 4. Distilled water (10 mL/kg, p.o.) to }\end{array}$ & & $\begin{array}{l}\text { CGRP-induced relaxation of } \\
\text { Prostaglandin F2 } \alpha \text {-induced } \\
\text { vasoconstriction in isolated } \\
\text { mesenteric vascular beds } \downarrow\end{array}$ \\
\hline & & & & & & 6 & $32(8 / 8 / 8 / 8)$ & sham-operated rats. & & $\begin{array}{l}\text { 125I-CGRP binding in isolated } \\
\text { mesenteric arteries } \downarrow\end{array}$ \\
\hline & & & & & & 7 & $38(10 / 9 / 10 / 9)$ & & & Plasma concentration of CGRP \\
\hline & & & & & & 8 & $29(7 / 7 / 8 / 7)$ & & & $\begin{array}{l}\text { Plasma concentration of } E_{2} \text { (no } \\
\text { sig); uterine weight (no sig) }\end{array}$ \\
\hline \multirow[t]{3}{*}{$\begin{array}{l}\text { Noguchi et al., } \\
2005[68]\end{array}$} & English, Japan & $\begin{array}{l}\text { Sprague-Dawley } \\
\text { rats }\end{array}$ & $\begin{array}{c}\text { GnRH } \\
\text { analog } \\
\text { Leupline (1 } \\
\mathrm{mg} / \mathrm{kg}) \\
\text { injection }\end{array}$ & 9 & $200-240$ & 9 & $\begin{array}{c}36 \\
(6 / 6 / 5 / 7 / 7 / 5)\end{array}$ & $\begin{array}{l}\text { 1. GFW }(100 \mathrm{mg} / \mathrm{kg}, \text { p.o.); } \\
\text { 2. GFW (300 mg/kg, p.o.); } \\
\text { 3. GFW (1,000 mg/kg, p.o.); } \\
\text { 4. E }(0.010 \mathrm{mg} / \mathrm{kg}, \text { s.c.); } \\
\text { 5. Distilled water }(10 \mathrm{~mL} / \mathrm{kg}, \text { p.o.) to injected rats; } \\
\text { 6. Distilled water }(10 \mathrm{~mL} / / \mathrm{kg}, \text { p.o.) to } \\
\text { sham-injected rats. }\end{array}$ & 14 & $\begin{array}{l}\text { CGRP-induced elevation of } \\
\text { skin temperature } \downarrow\end{array}$ \\
\hline & & & & & & & $\begin{array}{c}52 \\
(13 / 13 / 13 / 13)\end{array}$ & $\begin{array}{l}\text { 1. GFW }(1000 \mathrm{mg} / \mathrm{kg} \text {, p.o.); } \\
\text { 2. } \mathrm{E}_{2}(0.010 \mathrm{mg} / \mathrm{kg} \text {, s.c.); }\end{array}$ & & Plasma concentration of CGRP \\
\hline & & & & & & & $28(8 / 7 / 7 / 6)$ & $\begin{array}{l}\text { 3. Distilled water }(10 \mathrm{~mL} / \mathrm{kg}, \mathrm{p} \text {.o.) to injected rats; } \\
\text { 4. Distilled water } 10 \mathrm{~mL} / \mathrm{kg} \text {, p.o.) to } \\
\text { sham-injected rats. }\end{array}$ & & $\begin{array}{l}\text { Pituitary LH and FSH, plasma } \\
\mathrm{E}_{2} \text { and weights of uterus and } \\
\text { ovaries (no sig) }\end{array}$ \\
\hline
\end{tabular}

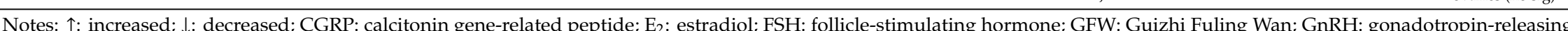
hormone; i.p.: intraperitoneal injection; LH: luteinizing hormone; NS: not specified; OVX: ovariectomized; p.o.: oral administration; s.c.: subcutaneous injection; sig: significance. 


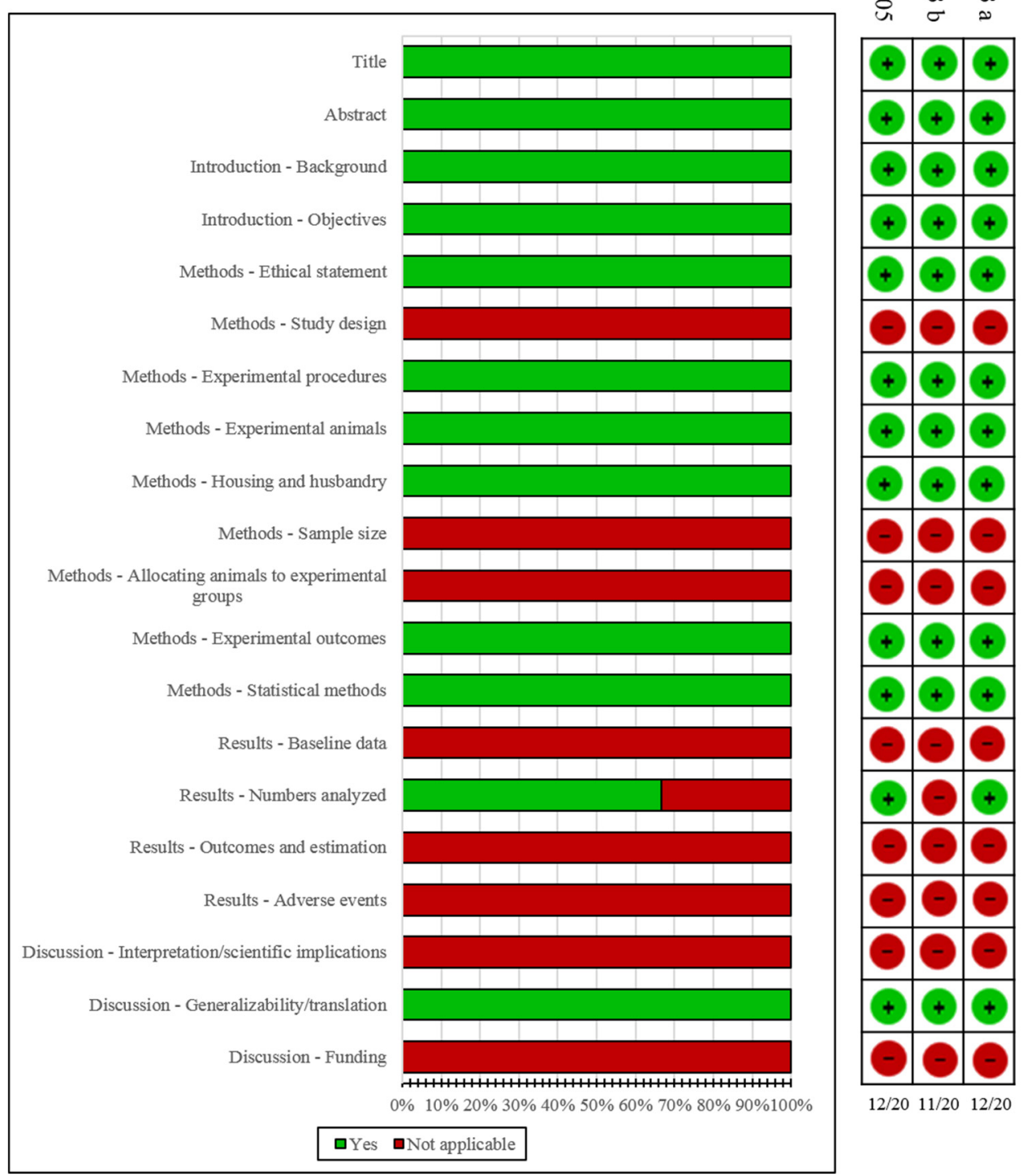

Figure 3. Quality assessment of included in vivo studies according to ARRIVE (Animal Research: Reporting of In Vivo Experiments) guideline.

One in vitro experimental study [69] utilized an ESR-dependent cell proliferation bioassay and an ESR-dependent reporter assay to investigate the potential estrogenic activity of GFW and its metabolites. The results demonstrated that GFW showed no estrogenic activity and low ER $\beta$-dependent estrogenic activity before or after metabolization with a concentration at $100 \mu \mathrm{g} / \mathrm{mL}$. Findings from two in vivo studies concluded that $\mathrm{E}_{2}$ could significantly reduce the levels of pituitary $\mathrm{LH}$ (luteinizing hormone) and FSH, as well as the weights of uterus and ovaries, whereas GFW did not influence those factors $[67,68]$. Thus, GFW did not confer estrogen activity on plasma and might exert pharmacodynamic effects against MHFs via other pathways. 


\section{Translation from Experimental Studies to Clinical Practice}

\subsection{Therapeutic Effects}

A well-designed RCT is considered as the gold standard when evaluating the effects of an intervention [70]. However, its limitations have triggered extensive discussion as RCTs may be unnecessary, inappropriate, inadequate, or even impossible in the clinic, and methodological problems can cause bias in the results [71-73]. Besides, CHM involves a holistic therapeutic approach. However, most of the published RCTs on CHM were designed to only investigate the therapeutic aspects (i.e., Chinese medicine intervention + western medicine diagnosis) instead of the holistic therapeutic approach (i.e., Chinese medicine intervention + Chinese medicine diagnosis) [74]. The importance of integration of various study designs was highlighted to accommodate the evaluation of various research questions and for the outcome of interest $[75,76]$. Therefore, the therapeutic effects of a range of study designs were systematically reviewed (Figure 4).

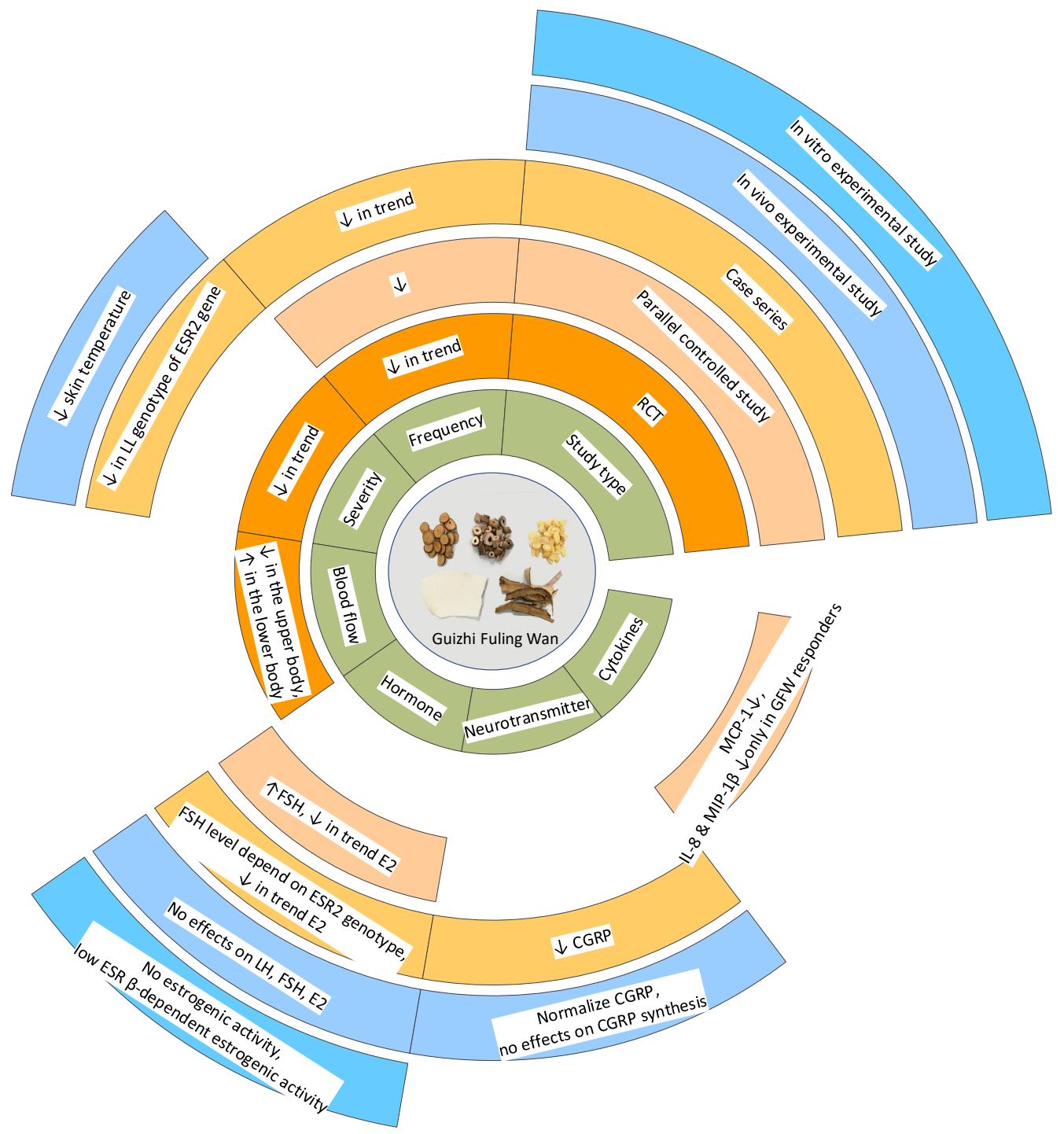

Figure 4. Therapeutic effects of Guizhi Fuling Wan on menopausal hot flushes across different types of studies. Notes: CGRP: calcitonin gene-related peptide; $\mathrm{E}_{2}$ : estradiol; ESR: estrogen receptor; FSH: follicle-stimulating hormone; 1IL: interleukin; LH: luteinizing hormone; LL: two long alleles; MIP: macrophage inflammatory protein; MCP: serum monocyte chemotactic protein; RCT: randomized controlled trial. 
As shown in Figure 4, the study types and outcome measures varied across all the included studies. Most outcomes were measured in different study designs. GFW demonstrated overall no statistically significant changes in the frequency and severity of MHFs between before and after treatment and no statistically significant differences between GFW and control groups. However, dose-dependent skin temperature changes were detected in both clinical [15] and experimental studies [69,70]. The effective dose suggested in the in vivo study was $1000 \mathrm{mg} / \mathrm{kg}[69,70]$, which was four times the dose in the RCT (12.5 g/day) when the participant weighed in at $50 \mathrm{~kg}$ [15]. Thus, more dose variation should be considered in further RCTs. The influence of GFW on FSH and $E_{2}$ was unclear based on the included studies due to the limitation of study design. Results of experimental studies indicated that GFW had no effects on LH, FSH, and $E_{2}$ in Sprague-Dawley rats and no estrogenic effects on the rat liver S9 fraction. However, case series studies pointed out that GFW might target a specific human ESR2 beta genotype. GFW was found to affect CGRP level in both case series and in vivo studies $[28,66,67,69]$. GFW had similar effects to $E_{2}$ on normalizing CGRP level but without affecting its synthesis in the dorsal root ganglia. Only peripheral blood flow and cytokines were reported in one study design, which should be further investigated in various studies.

\subsection{Potential Multi-Targeting Actions}

Three potential target proteins/cytokine could be proposed based on findings from the above-mentioned clinical and experimental studies: estrogen beta receptor, IL-8, and CGRP receptor.

There are significant associations between the ESR2 CA dinucleotide repeat length and other menopausal-related symptoms. For example, low bone mineral density has been reported previously in pre- and post-menopausal women $[31,77,78]$. This genetic variation was characterized by Tsukamoto and co-workers in 1998 [79]. Recently, ESR2 CA dinucleotide repeat length was reported to be closely related to clinical effects of GFW on MHFs and that it had better performance on participants with the LL genotype [29]. Women with the SS genotype had significantly higher values of circulation estrone concentration (highest) when compared to those harboring the LL genotype (lowest) after adjustment for other confounding factors, including age, hormone replacement status, and circulating levels of sex hormone-binding globulin [31]. Thus, genetic variation should be considered as an essential factor of using GFW for MHFs to achieve precision treatment. The prevalence of the SS and SL genotypes was approximately $47.79 \%(108 / 226)$ and $22.56 \%$ (51/226), respectively [31]. The sum of these two genotypes was much higher than that of the LL genotype $(29.65 \%, 67 / 226)$. Thus, patients without targeting genotype might contribute to the negative results in the RCT. It is suggested that future research focus on the three genotypes of ESR2 for precision medicine.

IL-8, also known as CXCL8, is a cytokine potentially involved in thermoregulation and was detected to be higher in premenopausal and menopausal women with MHFs than in those without MHFs [80,81]. GFW could significantly reduce concentrations of serum IL-8, whereas those in the non-responders were increased dramatically [27]. Thus, GFW might selectively influence the synthesis of IL-8. However, the underlying mechanism of this selective effect in different MHF sufferers is not fully understood.

CGRP is a widely expressed sensory neuropeptide, which plays a major role in modulating metabolism, inflammatory response, and blood pressure, as well as contributing to nerve development and function [82-86]. In the sympathetic nervous system, CGRP is believed to be the specific vasodilator responsible for MHFs [4,84]. Recently, Liang et al. [86] have determined the structure of the human CGRP receptor in complex with CGRP, with the Gs-protein heterotrimer at $3.3 \AA$ global resolution, which makes it possible for future investigation of the potential herb-target relationship between GFW and MHF-related target protein.

\subsection{Safety}

Inorganic elements that may have vital biological activities in the human body were traced in GFW, including calcium, sodium, magnesium with a dose over $300 \mu \mathrm{g} / \mathrm{g}$, followed by iron, manganese, 
and strontium with a dose over $10 \mu \mathrm{g} / \mathrm{g}$ [65]. The dosage of toxic inorganic elements detected in the included studies included beryllium, arsenic, cadmium, antimony, mercury, thallium, and bismuth, which were under $0.03 \mu \mathrm{g} / \mathrm{g}$ and were lower than the maximum limit level listed in the Chinese Pharmacopoeia [65]. GFW is believed to be a safe herbal product as only a small number of minor gastrointestinal symptoms were reported as adverse effects: two studies reported adverse events, including diarrhea $(n=15)$ [15] and abnormal feeling in the gastrointestinal tract $(n=2)$ [27]. They were the main causes leading to drop out. Additionally, an in vivo study on Sprague-Dawley rats indicating that GFW was not carcinogenic after a continuous 24-month intervention [87]. Results from another in vitro experiment [68] on estrogen-dependent human breast cancer (MCF-7) cells showed that GFW at concentrations of 10.6-10.4 mg/mL did not activate the proliferation of MCF-7 cell, which suggested that GFW did not exhibit estrogen activity. Therefore, GFW might be a potential therapeutic option for MHFs in women who are undergoing breast cancer treatment.

\subsection{Implications for Future Research}

In total, 169 compounds from GFW and three potential target proteins/cytokine (estrogen beta receptor, IL-8, and CGRP receptor) were identified based on findings from the above-mentioned clinical and experimental studies. However, at this stage, a clear understanding of their interactions could not be obtained based on current literature. The bioactivities of the phytochemicals and their mechanisms of actions on MHF were not fully investigated. Although quality control is improving during the last two decades, evidenced by the increased quantitative index from one in the 2005 edition to two or three in the 2015 edition of the Chinese Pharmacopeia [17], not all the herbal ingredients have a quantitative index. The clinical effects of $\mathrm{CHM}$ are often regarded as the result of the multi-targeting interaction of various phytochemical compounds. Thus, more research on the mechanism of actions of CHM on a condition at the molecular level (ligand-target interaction) and the therapeutic dose of the bioactive compounds are warranted.

\section{Conclusions}

This study identified 169 compounds from GFW pills, tablets, and capsules. Therapeutic effects, including frequency and severity of MHFs, peripheral blood flow, hormones, neurotransmitter, and cytokines, were not consistent across different study types. ESR2 with genetic variation, CGRP receptor, and IL- 8 were identified to be related to the mechanisms of actions of GFW on MHFs. The differences in therapeutic effects could be potentially due to dose variations among clinical and experimental studies and ESR2 gene differences in rats and homo species. Further clinical and experimental studies, as well as biochemical investigation on the mechanisms of actions of GFW, are warranted. It is recommended that the dose-dependent effects and ESR2 expression at the participant recruitment stage are considered in future investigation.

Author Contributions: Conceptualization, M.L., A.W.H.Y., and A.H.; Formal Analysis, M.L., A.W.H.Y., A.H., and H.L.; Data curation, M.L., A.W.H.Y., A.H., and H.L.; Writing-Original Draft Preparation, M.L.; Writing-Review and Editing, A.W.H.Y., A.H., and H.L.; Visualization, M.L. and H.L.; Supervision, A.W.H.Y., and A.H.; Project Administration, M.L., A.W.H.Y., and A.H.

Funding: This research received no external funding.

Acknowledgments: We thank Mathew Guo from the Nanjing University of Chinese Medicine and Chunlan Wang from the Ocean University of China for their assistance in searching the SinoMed and CNKI database.

Conflicts of Interest: The authors declare no conflict of interest.

\section{References}

1. Freeman, E.W.; Sherif, K. Prevalence of hot flushes and night sweats around the world: A systematic review. Climacteric 2007, 10, 197-214. [CrossRef] [PubMed] 
2. Berek, J.S.; Novak, E. Berek Novak's Gynecology, 15th ed.; Wolters Kluwer Health/Lippincott Williams \& Wilkins: Philadelphia, PA, USA, 2012.

3. Jha, U.P. Hot flushes. In Differential Diagnosis in Obstetrics and Gynaecology: An a-z; Hollingworth, T., Ed.; Hodder Arnold: London, UK, 2008; pp. 43-48.

4. Gupta, P.; Harte, A.L.; da Silva, N.F.; Khan, H.; Barnett, A.H.; Kumar, S.; Sturdee, D.W.; McTernan, P.G. Expression of calcitonin gene-related peptide, adrenomedullin, and receptor modifying proteins in human adipose tissue and alteration in their expression with menopause status. Menopause 2007, 14, 1031-1038. [CrossRef] [PubMed]

5. Chakravarti, S.; Collins, W.P.; Newton, J.R.; Oram, D.H.; Studd, J.W.W. Endocrine changes and symptomatology after oophorectomy in premenopausal women. BJOG 1977, 84, 769-775. [CrossRef]

6. Schuiling, K.D.; Likis, F.E. Women's Gynecologic Health, 3rd ed.; Jones \& Bartlett Learning: Burlington, MA, USA, 2017.

7. Freedman, R.R.; Krell, W. Reduced thermoregulatory null zone in postmenopausal women with hot flashes. AJOG 1999, 181, 66-70. [CrossRef]

8. Freedman, R.R. Biochemical, metabolic, and vascular mechanisms in menopausal hot flashes. Fertil. Steril. 1998, 70, 332-337. [CrossRef]

9. Berendsen, H.H.G. The role of serotonin in hot flushes. Maturitas 2000, 36, 155-164. [CrossRef]

10. Mintziori, G.; Lambrinoudaki, I.; Goulis, D.G.; Ceausu, I.; Depypere, H.; Erel, C.T.; Pérez-López, F.R.; Schenck-Gustafsson, K.; Simoncini, T.; Tremollieres, F. EMAS position statement: Non-hormonal management of menopausal vasomotor symptoms. Maturitas 2015, 81, 410-413. [CrossRef]

11. Wnuk, A.; Korol, D.L.; Erickson, K.I. Estrogens, hormone therapy, and hippocampal volume in postmenopausal women. Maturitas 2012, 73, 186-190. [CrossRef]

12. National Guideline Clearinghouse. Menopause: Diagnosis and Management. Available online: https://www.guideline.gov/summaries/summary/49904/menopause-diagnosis-and-management? q=menopausal (accessed on 8 November 2017).

13. Peng, W.; Adams, J.; Hickman, L.; Sibbritt, D.W. Longitudinal analysis of associations between women's consultations with complementary and alternative medicine practitioners/use of self-prescribed complementary and alternative medicine and menopause-related symptoms, 2007-2010. Menopause 2016, 23, 74-80. [CrossRef]

14. Ushiroyama, T.; Ikeda, A.; Sakuma, K.; Ueki, M. Comparing the effects of estrogen and an herbal medicine on peripheral blood flow in post-menopausal women with hot flashes: Hormone replacement therapy and Gui-Zhi-Fu-Ling-Wan, a Kampo medicine. AJCM 2005, 33, 259. [CrossRef]

15. Plotnikoff, G.A.; Watanabe, K.; Torkelson, C.; La Valleur, J.; Radosevich, D.M.; Plotnikoff, G.A.; Watanabe, K.; Torkelson, C.; La Valleur, J.; Radosevich, D.M. The TU-025 keishibukuryogan clinical trial for hot flash management in postmenopausal women: Results and lessons for future research. Menopause 2011, 18, 886-892. [CrossRef]

16. Zhang, Z.J. Gui Zhi Fu Ling Wan. In Jin Guì Yào Lüè Essential Prescriptions of the Golden Cabinet: Translation and Commentaries; Wiseman, N., Wilms, S., Eds.; Paradigm Publications: Taos, NM, USA, 2013; pp. $493-497$.

17. Chinese Pharmacopoeia Commission. Chinese Pharmacopoeia, 2015 ed.; China Medical Science Press: Beijing, China, 2015.

18. ClinicalTrials, ClinicalTrials.gov. Effect of KYG0395 on Primary Dysmenorrhea. Available online: https: //clinicaltrials.gov/ct2/show/study/NCT01588236?term=KYG0395\&rank=1 (accessed on 6 August 2019).

19. Ni, H.; Matsumoto, T.; Watanabe, J.; Makino, T. Inhibitory Effect of Japanese Traditional Kampo Formula Frequently Prescribed in Gynecological Clinics on CYP3A4. eCAM 2018, 2018, e4259603. [CrossRef]

20. Lee, H.S.; Shin, H.J.; Cho, M.; Lee, S.H.; Oh, D.S. Inhibitory effects of Kampo medicines, Keishibukuryogan and Shakuyakukanzoto, on the substrate uptake activities of solute carrier organic anion transporters. JPS 2018, 138, 279-283. [CrossRef]

21. Furue, M.; Fuyuno, Y.; Mitoma, C.; Uchi, H.; Tsuji, G. Therapeutic agents with AHR inhibiting and NRF2 activating activity for managing chloracne. Antioxidants 2018, 7, 90. [CrossRef] [PubMed]

22. Terauchi, M.; Hiramitsu, S.; Akiyoshi, M.; Owa, Y.; Kato, K.; Obayashi, S.; Matsushima, E.; Kubota, T. Effects of three Kampo formulae: Tokishakuyakusan (TJ-23), Kamishoyosan (TJ-24), and Keishibukuryogan (TJ-25) on Japanese peri- and postmenopausal women with sleep disturbances. Arch. Gynecol. Obstet. 2010, 284, 1-9. [CrossRef] [PubMed] 
23. Terauchi, M.; Akiyoshi, M.; Owa, Y.; Kato, K.; Obayashi, S.; Kubota, T. Effects of the Kampo medication keishibukuryogan on blood pressure in perimenopausal and postmenopausal women. Int. J. Gynaecol. Obstet. 2011, 114, 149-152. [CrossRef]

24. Higgins, J.P.T.; Green, S. Cochrane Handbook for Systematic Reviews of Interventions; Wiley Blackwell: Chichester, UK; Hoboken, NJ, USA, 2011.

25. Sterne, J.A.C.; Hernán, M.A.; Reeves, B.C.; Savović, J.; Berkman, N.D.; Viswanathan, M.; Henry, D.; Altman, D.G.; Ansari, M.T.; Boutron, I. ROBINS-I: A tool for assessing risk of bias in non-randomised studies of interventions. Br. Med. J. 2016, 355, i4919. [CrossRef]

26. Yang, A.W.; Li, C.G.; Da Costa, C.; Allan, G.; Reece, J.; Xue, C.C. Assessing quality of case series studies: Development and validation of an instrument by herbal medicine CAM researchers. J. Altern. Complement. Med. 2009, 15, 513-522. [CrossRef]

27. Yasui, T.; Matsui, S.; Yamamoto, S.; Uemura, H.; Tsuchiya, N.; Noguchi, M.; Yuzurihara, M.; Kase, Y.; Irahara, M. Effects of Japanese traditional medicines on circulating cytokine levels in women with hot flashes. Menopause 2011, 18, 85-92. [CrossRef]

28. Chen, J.T.; Shiraki, M. Menopausal hot flash and calciotonin gene-related peptide; effect of Keishi-bukuryo-gan, a kampo medicine, related to plasma calciotonin gene-related peptide level. Maturitas 2003, 45, 199-204. [CrossRef]

29. Namiki, T.; Sato, H.; Matsumoto, Y.; Kakikura, H.; Ueno, K.; Chino, A.; Okamoto, H.; Hisanaga, A.; Kaneko, A.; Kita, T.; et al. Identification of a predictive biomarker for the beneficial effect of Keishibukuryogan, a Kampo (Japanese traditional) medicine, on patients with climacteric syndrome. eCAM 2014, 2014, e962109. [CrossRef]

30. Westberg, L.; Baghaei, F.; Rosmond, R.; Hellstrand, M.; Landen, M.; Jansson, M.; Holm, G.; Bjorntorp, P.; Eriksson, E. Polymorphisms of the androgen receptor gene and the estrogen receptor beta gene are associated with androgen levels in women. J. Clin. Endocrinol. Metab. 2001, 86, 2562-2568.

31. Scariano, J.K.; Simplicio, S.G.; Montoya, G.D.; Garry, P.J.; Baumgartner, R.N. Estrogen receptor $\beta$ dinucleotide (CA) repeat polymorphism is significantly associated with bone mineral density in postmenopausal women. Calcif. Tissue Int. 2004, 74, 501-508. [CrossRef]

32. Sturdee, D.W.; Hunter, M.S.; Maki, P.M.; Gupta, P.; Sassarini, J.; Stevenson, J.C.; Lumsden, M.A. The menopausal hot flush: A review. Climacteric 2017, 20, 296-305. [CrossRef]

33. Dassault Systèmes BIOVIA. BIOVIA Draw; Dassault Systèmes: San Diego, CA, USA, 2019.

34. Hanwell, M.D.; Curtis, D.E.; Lonie, D.C.; Vandermeersch, T.; Zurek, E.; Hutchison, G.R. Avogadro: An advanced semantic chemical editor, visualization, and analysis platform. J. Cheminform. 2012, 4, 17. [CrossRef]

35. Dassault Systèmes BIOVIA. Discovery Studio Visualizer; Dassault Systèmes: San Diego, CA, USA, 2019.

36. Kilkenny, C.; Browne, W.J.; Cuthill, I.C.; Emerson, M.; Altman, D.G. Improving bioscience research reporting: The ARRIVE guidelines for reporting animal research. PLoS Biol. 2010, 8, e1000412. [CrossRef]

37. Zhang, Y.; Cheng, Y.; Liu, Z.; Ding, L.; Qiu, T.; Chai, L.; Qiu, F.; Wang, Z.; Xiao, W.; Zhao, L.; et al. Systematic screening and characterization of multiple constituents in Guizhi Fuling capsule and metabolic profiling of bioactive components in rats using ultra-high-performance liquid chromatography/quadrupole-time-of-flight mass spectrometry. J. Chromatogr. B 2017, 1061, 474-486. [CrossRef]

38. Feng, C.P. Determination of cinnamic acid, cinnamaldehyde and paeonol in Guizhi Fuling pills by HPLC. Guid. J. Tradit. Chin. Med. Pharm. 2009, 15, 76-78.

39. Hua, J.; $\mathrm{Wu}, \mathrm{C}$. Determination of cinnamic acid, cinnamaldehyde and paeonol in Guizhi Fuling capsules by HPLC. Anhui Med. Pharm. J. 2006, 10, 30-31.

40. Yang, Q.; Guo, Y.R. Simultaneous determination of cinnamaldehyde and paeonol in Guizhi Fuling capsules by SPE-HPLC. Chin. Pharm. 2010, 13, 1312-1314.

41. He, Y.M.; Lin, X.; Wang, X.; Li, J.C.; Huang, W.Z.; Xiao, W. Determination of dissolution of eight active ingredients in Guizhi Fuling capsules by UPLC. Chin. J. Exp. Tradit. Med. Formulae 2018, 24, 14-19.

42. Xie, X.; Zhang, H.D.; Wen, J.H.; Song, Y.L.; Wang, X.J.; Zhao, W.W.; Huang, W.Z.; Wang, Z.Z.; Xiao, W. Study on chemical constituents of Guizhi Fuling capsules (VI). Chin. Tradit. Herb. Drugs 2016, 47, 3795-3797.

43. Li, W.S.; Huang, L.L.; Li, Z.J.; Tang, M.H.; Guo, K. Optimization of extraction solvent in the chemical composition analysis of Guizhi Fuling capsules by HPLC-ESI-QTOF/MS. Chin. J. Bioproc. E 2016, 14, 59-64. 
44. Zhang, Y.H.; Zhang, D.W.; Meng, Z.Q.; Liu, L.Y.; Jin, Y. Rapid determination of paeoniflorin, paeonol, amygdalin and cinnamic acid in Guizhi Fuling capsules by online two-dimensional liquid chromatography. Zhongguo Zhong Yao Za Zhi 2013, 38, 4088-4093.

45. Sui, Y.; Zhao, L.S.; Wang, Z.Z.; Zhao, Y.T.; Xiao, W.; Xiong, Z.L. Simultaneous determination of seven bioactive components in Guizhi Fuling capsule by microwave-assisted extraction combined with ultra performance liquid chromatography tandem mass spectrometry. Nat. Prod. Res. 2016, 30, 354-357. [CrossRef]

46. Yin, Q.W.; Wang, J.L.; Yang, S.D.; Huang, W.Z.; Xiao, W. Analysis of $\alpha$-linolenic acid, linoleic acid and oleic acid in Guizhi Fuling capsules. Pharm. Clin. Res. 2016, 24, 110-112.

47. Zhang, Q.; Chen, M.L.; Sun, Y.L.; Zhang, Y.; Gao, X.Y. Simultaneous measurement of amygdalin, paeonol and paeoniflorin in Guizhi Fuling tablets by HPLC. World Latest Med. Inf. 2017, 17, 113-114.

48. Jin, Q.X.; Huang, Y.F. Determination of amygdalin in ethanol treatment of Guizhi Fuling capsules by HPLC. Chin. Arch. Tradit. Chin Med. 2012, 30, 381-382.

49. Wang, H.Y.; Huang, Y.F. ANOVA for optimization of amygdalin leaching rate in Guizhi Fuling capsules. Zhongguo Zhong Yao Za Zhi 2011, 29, 2700-2701.

50. Fang, L.Q. Simultaneous determination of paeonol, paeoniflorin and amygdalin in Guizhi Fuling capsules by HPLC. J. North Pharm. 2015, 12, 5-6.

51. Liao, Z.G.; Jiang, Q.Y.; Liang, X.L.; Ping, Q.N. Comparative study on the dissolution of three active ingredients in Guizhi Fuling capsules in vitro. Chin. Tradit. Patent Med. 2008, 30, 1141-1144.

52. Ma, T.T.; Chen, X.H.; Lin, X.Y.; Guo, D.A.; Bi, K.S. Simultaneous determination of three components in Guizhi Fuling capsules by RP-HPLC. J. Shenyang Pharm. Univ. 2010, 27, 216-219.

53. Wang, X.J.; Xie, X.; Luo, X.; Zhao, W.W.; Huang, W.Z.; Wang, Z.Z.; Xiao, W. Study on chemical constituents of Guizhi Fuling capsules (V). Chin. Tradit. Herb. Drugs 2015, 46, 812-816.

54. Wang, H.Q.; Liu, C.; Fang, L.H.; Wang, Z.Z.; Xiao, W.; Chen, R.Y. Study on chemical constituents of Guizhi Fuling capsules (IV). Chin. Tradit. Herb. Drugs 2013, 44, 1386-1390.

55. Yang, P.F.; Wang, Z.Z.; Wang, H.Q.; Li, J.C.; Chen, R.Y. Study on chemical constituents of Guizhi Fuling capsules (III). Chin. Tradit. Herb. Drugs 2012, 43, 463-466.

56. Shi, X.Q. Determination of paeonol in Guizhi Fuling capsules by gas chromatography. Guangzhou Chem. Ind. 2015, 43, 145-146, 173.

57. Pan, H.P.; Chen, Y.; Fu, C.L. Determination of paeonol in Guizhi Fuling capsules by HPLC. Zhongguo Zhong Yao Za Zhi 2002, 27, 34-36.

58. Mao, J.X. Determination of paeonol in Guizhi Fuling capsules by HPLC. Lishizhen Med. Mater. Med. Res. 2009, 20, 2112.

59. Wu, X.H.; Fang, H.; Pu, C.Y.; Yang, D.X.; Wang, F.S.; He, L.W.; Zhang, N. Study of Guizhi Fuling pills using HPLC Fingerprint. J. Anal. Sci. 2014, 30, 100-102.

60. Niu, S.L.; Guo, Y.L.; Liu, X.; Zhang, X.N. Determination of paeoniflorin in Guizhi Fuling capsules by HPLC. Herald Med. 2004, 23, 863.

61. Ma, Y.; Zheng, W.R.; Wang, Z.Z.; Xiao, W.; Huang, W.Z.; Zhang, Y.W. UPLC/Q-TOF-MS fingerprint of triterpenic acids in Guizhi Fuling capsules. Chin. Tradit. Herb. Drugs 2019, 50, 626-631.

62. Yang, P.F.; Li, B.M.; Wang, Z.Z.; Xiao, W.; Chen, R.Y. Simultaneous determination of four barium tridecanoic acids in Guizhi Fuling capsules by HPLC. Chin. Tradit. Herb. Drugs 2016, 47, 3215-3218.

63. Wu, L.-F.; Wang, K.-F.; Mao, X.; Liang, W.-Y.; Chen, W.-J.; Li, S.; Qi, Q.; Cui, Y.-P.; Zhang, L.-Z. Screening and analysis of the potential bioactive components of Poria cocos (Schw.) wolf by HPLC and HPLC-MSn with the aid of chemometrics. Molecules 2016, 21, 227. [CrossRef]

64. Ma, Y.; Li, J.C.; Huang, W.Z.; Wang, Z.Z.; Xiao, W.; Song, Y.L.; Zhang, Y.W. Simultaneous determination of six triterpenoids in Guizhi Fuling capsules by UPLC-MS/MS. Zhongguo Zhong Yao Za Zhi 2017, 42, 1-12.

65. Kang, Y.; Liu, X.H.; Wang, B. Analysis of inorganic elements in Guizhi Fuling capsules based on ICP-MS. Chin. Tradit. Herb. Drugs 2018, 49, 3292-3297.

66. Noguchi, M.; Ikarashi, Y.; Yuzurihara, M.; Kase, Y.; Takeda, S.; Aburada, M. Effects of 17b-estradiol and the japanese herbal medicine keishi-bukuryo-gan on the release and synthesis of calcitonin gene-related peptide in ovariectomized rats. J. Pharmacol. Sci. 2003, 93, 80-86. [CrossRef] 
67. Noguchi, M.; Ikarashi, Y.; Yuzurihara, M.; Kase, Y.; Chen, J.T.; Takeda, S.; Aburada, M.; Ishige, A. Effects of the Japanese herbal medicine Keishi-bukuryo-gan and 17beta-estradiol on calcitonin gene-related peptide-induced elevation of skin temperature in ovariectomized rats. J. Endocrinol. 2003, 176, 359-366. [CrossRef]

68. Noguchi, M.; Ikarashi, Y.; Yuzurihara, M.; Kase, Y.; Watanabe, K.; Plotnikoff, G.A.; Takeda, S.; Aburada, M. Skin temperature rise induced by calcitonin gene-related peptide in gonadotropin-releasing hormone analogue-treated female rats and alleviation by Keishi-bukuryo-gan, a Japanese herbal medicine. Life Sci. 2005, 76, 2079-2090. [CrossRef]

69. Wang, Z.; Kanda, S.; Shimono, T.; Enkh-Undraa, D.; Nishiyama, T. The in vitro estrogenic activity of the crude drugs found in Japanese herbal medicines prescribed for menopausal syndrome was enhanced by combining them. BMC Complement. Altern. Med. 2018, 18, 107. [CrossRef]

70. Akobeng, A. Understanding randomised controlled trials. Arch. Dis. Child. 2005, 90, 840-844. [CrossRef]

71. Earl-Slater, A. The Handbook of Clinical Trials and Other Research; Radcliffe Medical: Abingdon, UK, 2002.

72. Greenhalgh, T.A. How to Read a Paper: The Basics of Evidence-Based Medicine, 5th ed.; John Wiley \& Sons: Chichester, UK; Hoboken, NJ, USA, 2014.

73. Liu, J.P. Clinical Research Methodology for Evidence-Based Chinese Medicine; China Agriculture Press: Beijing, China, 2006.

74. Liu, J.P. The system of evidence levels in Chinese Medicine clinical trial. In Clinical Research Methodology for Evidence-Based Chinese Medicine; People's Medicine Publishing House: Beijing, China, 2006; pp. 11-31.

75. Peinemann, F.; Tushabe, D.A.; Kleijnen, J. Using multiple types of studies in systematic reviews of health care interventions-a systematic review. PLoS ONE 2013, 8, e85035. [CrossRef]

76. Vandenbroucke, J.P. Observational research, randomised trials, and two views of medical science. PLoS Med. 2008, 5, e67. [CrossRef]

77. Lau, H.H.; Ho, A.Y.; Luk, K.D.; Kung, A.W. Estrogen receptor beta gene polymorphisms are associated with higher bone mineral density in premenopausal, but not postmenopausal southern Chinese women. Bone 2002, 31, 276-281. [CrossRef]

78. Ogawa, S.; Hosoi, T.; Shiraki, M.; Orimo, H.; Emi, M.; Muramatsu, M.; Ouchi, Y.; Inoue, S. Association of estrogen receptor beta gene polymorphism with bone mineral density. Biochem. Biophys. Res. Commun. 2000, 269, 537-541. [CrossRef]

79. Tsukamoto, K.; Inoue, S.; Hosoi, T.; Orimo, H.; Emi, M. Isolation and radiation hybrid mapping of dinucleotide repeat polymorphism at the human estrogen receptor beta locus. J. Hum. Genet. 1998, 43, 73-74. [CrossRef]

80. Yasui, T.; Uemura, H.; Tomita, J.; Miyatani, Y.; Yamada, M.; Kuwahara, A.; Matsuzaki, T.; Maegawa, M.; Tsuchiya, N.; Yuzurihara, M. Association of interleukin-8 with hot flashes in premenopausal, perimenopausal, and postmenopausal women and bilateral oophorectomized women. J. Clin. Endocrinol. Metab. 2006, 91, 4805-4808. [CrossRef]

81. Malutan, A.; Costin, N.; Duncea, I.; Pepene, C.; Mihu, D.; Rada, M. Interleukin-8 and vasomotor symptoms in natural and surgically induced menopause. Acta Endocrinol. 2013, 9, 133-144. [CrossRef]

82. Walker, C.S.; Li, X.; Whiting, L.; Glyn-Jones, S.; Zhang, S.; Hickey, A.J.; Sewell, M.A.; Ruggiero, K.; Phillips, A.R.; Kraegen, E.W.; et al. Mice lacking the neuropeptide alpha-calcitonin gene-related peptide are protected against diet-induced obesity. Endocrinology 2010, 151, 4257-4269. [CrossRef]

83. Dickerson, I.M.; Bussey-Gaborski, R.; Holt, J.C.; Jordan, P.M.; Luebke, A.E. Maturation of suprathreshold auditory nerve activity involves cochlear CGRP-receptor complex formation. Physiol. Rep. 2016, 4, e12869. [CrossRef]

84. Russell, F.A.; King, R.; Smillie, S.J.; Kodji, X.; Brain, S.D. Calcitonin gene-related peptide: Physiology and pathophysiology. Physiol. Rev. 2014, 94, 1099-1142. [CrossRef]

85. Hay, D.L.; Garelja, M.L.; Poyner, D.R.; Walker, C.S. Update on the pharmacology of calcitonin/CGRP family of peptides: IUPHAR Review 25. Br. J. Pharmacol. 2018, 175, 3-17. [CrossRef] 
86. Liang, Y.-L.; Khoshouei, M.; Deganutti, G.; Glukhova, A.; Koole, C.; Peat, T.S.; Radjainia, M.; Plitzko, J.M.; Baumeister, W.; Miller, L.J.; et al. Cryo-EM structure of the active, Gs-protein complexed, human CGRP receptor. Nature 2018, 561, 492-497. [CrossRef]

87. Kanitani, M.; Nishimura, N.; Edamoto, H.; Kase, Y. Keishibukuryogan is not carcinogenic in Sprague-Dawley rats. J. Toxicol. Pathol. 2016, 29, 103-110. [CrossRef]

(C) 2019 by the authors. Licensee MDPI, Basel, Switzerland. This article is an open access article distributed under the terms and conditions of the Creative Commons Attribution (CC BY) license (http://creativecommons.org/licenses/by/4.0/). 